\title{
L'invariant de Suslin en caractéristique positive
}

\begin{abstract}
Résumé
Pour une $k$-algèbre simple centrale $A$ d'indice inversible dans $k$, Suslin a défini un invariant cohomologique de $\mathbf{S K}_{1}(A)$ [Sus2]. Dans ce texte, nous généralisons cet invariant à toute $k$-algèbre simple centrale par un relèvement de la caractéristique positive à la caractéristique 0 . Pour pouvoir définir cet invariant, on a besoin des groupes de cohomologie des différentielles logarithmiques de Kato [Kat1].
\end{abstract}

\section{Abstract}

For a central simple $k$-algebra $A$ with $\operatorname{ind}_{k}(A) \in k^{\times}$, Suslin defined a cohomological invariant for $\mathbf{S K}_{1}(A)$ Sus2]. In this text, we generalise his invariant to any central simple $k$-algebra using a lift from positive characteristic to characteristic 0 . To be able to define the invariant, we use Kato's cohomology of logarithmic differentials [Kat1].

\section{Introduction}

Soient $k$ un corps, $A$ une $k$-algèbre simple centrale et $\mathbf{S L}_{1}(A)$ le groupe algébrique linéaire usuel que nous considérons comme foncteur, donc défini pour chaque extension de corps $F$ de $k$ par

$$
\mathrm{SL}_{1}(A)(F)=\operatorname{SL}_{1}\left(A \otimes_{k} F\right)=\left\{a \in A \otimes_{k} F \mid \operatorname{Nrd}_{A \otimes_{k} F / F}(a)=1\right\} .
$$

Soit de plus $\mathbf{S K}_{1}(A)$ le foncteur en groupes qui associe à chaque extension de corps $F$ de $k$ le groupe de Whitehead réduit de $A_{F}:=A \otimes_{k} F$; i.e.

$$
\operatorname{SK}_{1}(A)(F)=\operatorname{SK}_{1}\left(A_{F}\right) \cong \operatorname{SL}_{1}\left(A_{F}\right) /\left[A_{F}^{\times}, A_{F}^{\times}\right] .
$$

Puisque $\mathbf{S K}_{1}(A)$ est donc lié au groupe algébrique linéaire $\mathbf{S L}_{1}(A)$, il vaut la peine de l'etudier, certainement parce que Platonov nous a rassuré qu'il n'est pas forcément trivial [Pla, Thm. 5.19]. La question de la trivialité a été indépendamment posée par Tannaka et Artin en 1943. Pendant plus de 30 années, on a essayé de la prouver - le problème de TannakaArtin [NM, Wan]. $\mathbf{S K}_{1}(A)$ est quand-même trivial si $\operatorname{ind}_{k}(A)$ est sans facteurs carrés (Théorème de Wang [Wan]). Suslin a conjecturé la réciproque [Sus1]. Récemment, Merkurjev a démontré que cette conjecture vaut si $4 \mid \operatorname{ind}_{k}(A)$ [Mer2], et Rehman-TikhonovYanchevskiı̌ ont démontré qu'il suffit de démontrer la conjecture pour des algèbres à division cycliques [RTY, Thm. 0.19].

Adresse : Tim Wouters, K.U.Leuven, Departement Wiskunde, Celestijnenlaan 200B bus 2400, B-3001 Leuven, Belgique - tim@wouters.in Classification (AMS) par sujet 2010 : 19B99 (12G05, 16K50, 17C20)

Mots clés : Groupe de Whitehead réduit - Modules de cycles - Invariants cohomologiques 
Une façon d'étudier $\mathbf{S K}_{1}(A)$ est de construire des invariants cohomologiques. Inspiré par Platonov [Pla], Suslin a construit un invariant cohomologique de $\mathbf{S K}_{1}(A)$ dans le cas où $n=\operatorname{ind}_{k}(A) \in k^{\times}$[Sus2]. Si $[A] \in H^{2}\left(k, \mu_{n}\right)$ est la classe de Brauer de $A$, l'invariant de Suslin est un morphisme fonctoriel en le corps (la fonctorialité est en effet inhérente à la définition d'un invariant) :

$$
\rho_{\mathrm{Sus}, A}: \mathbf{S K}_{1}(A)(k) \rightarrow H^{4}\left(k, \mu_{n}^{\otimes 3}\right) /\left(H^{2}\left(k, \mu_{n}^{\otimes 2}\right) \cup[A]\right) .
$$

Pour un aperçu plus détaillé de cette histoire, voir [Gil2, §2]. La conjecture de BlochKato, récemment démontrée par Voevodsky-Rost-Weibel [BK, Voe, Ros2, Wei], donne que le symbole galoisien $h_{n, k}^{i}: K_{i}^{M}(k) / n K_{i}^{M}(k) \rightarrow H^{i}\left(k, \mu_{n}^{\otimes i}\right)$ décrit les $K$-groupes de Milnor en termes de la cohomologie galoisienne $(i \geq 0)$. On sait donc réécrire l'image de $\rho_{\text {Sus }}$ avec une structure de $K_{n}^{M}(k)$-module qui est induite par le symbole galoisien.

Le but de ce texte est de généraliser l'invariant de Suslin pour toute $k$-algèbre simple centrale, utilisant qu'il existe pour toute algèbre simple centrale en caractéristique 0 . Si $k$ est un corps de caractéristique $p>0$, on le considère comme corps résiduel d'un anneau $R$ complet de valuation discrète $v$ avec corps des fractions $K$ de caractéristique 0 . On sait relever $A$ en une $R$-algèbre d'Azumaya $B$ tel que $B \otimes_{R} k \cong A$. Alors $B_{K}:=B \otimes_{R} K$ devient une $K$-algèbre simple centrale, et Suslin a donc construit un invariant cohomologique de $\mathbf{S K}_{1}\left(B_{K}\right)$. Vu qu'un résultat de Platonov [Pla, Cor. 3.13] induit un isomorphisme $\mathbf{S K}_{1}\left(B_{K}\right)(K) \cong \mathbf{S K}_{1}(A)(k)$, on peut essayer d'en déduire des invariants cohomologiques de $\mathbf{S K}_{1}(A)$. La subtilité ici est que l'isomorphisme de Platonov se déroule au niveau des groupes, et pas au niveau des foncteurs, ce qui cause quand même quelques soucis. De plus, la définition dépend du choix de $R$. Heureusement, on sait passer outre en utilisant des anneaux de Cohen.

On commence par le cas où car $(k)>0$ et l'indice de $A$ est inversible dans $k$ (le cas modéré). L'invariant de Suslin est en effet déjà défini dans ce cas, mais le relèvement permet aussi de construire un invariant en caractéristique positive à partir de tout invariant de $\mathbf{S K}_{1}\left(B_{K}\right)$ (en caractéristique 0) du même type. De plus, cela sera un bon échauffement pour le cas sauvage. Avant d'établir le relèvement, on décrit les groupes de cohomologie utilisés (Section 2). On les insère dans le formalisme des modules de cycles de Rost [Ros1] afin de pouvoir utiliser une caractérisation très utile des invariants de Merkurjev Mer1, Lem. 2.1, Thm. 2.3]. Puis, on a tous les ingrédients en vue de relever les invariants (Section 3).

Après ce relèvement, on continue au cas où l'indice de $A$ peut être non inversible dans $k$ (le cas sauvage). Afin de construire un invariant, il faut utiliser les groupes de cohomologie de différentielles logarithmiques de Kato [Kat1] qui généralisent la cohomologie galoisienne. Eux aussi sont servis d'une structure de $K_{n}^{M}(k)$-modules comme les groupes de cohomologie galoisienne ordinaires. Un résultat de Kahn [Kah] nous fournit le dernier ingrédient pour pouvoir effectuer un relèvement et de telle façon obtenir l'invariant désiré (Section 4). Utilisant la validité de la conjecture de Suslin pour les biquaternions, nous pouvons nous rassurer que cet invariant relevé est non trivial. Nous finissons ce papier avec quelques remarques : entre autre quelques considérations sur la conjecture de Suslin au regard des résultats obtenus.

Notations - Fixons quelques notations durant tout ce texte.

- Pour un corps $k$, on note $k_{s}$ une clôture séparable et $\Gamma_{k}=\operatorname{Gal}\left(k_{s} / k\right)$ le groupe de Galois absolu. 
- Pour un entier $m>0$ et un corps $k$, on note le $\Gamma_{k}$-module des racines $m$-ièmes d'unité de $k_{s}$ par $\mu_{m}$.

- Les groupes de cohomologies utilisés -sauf mention expresse- sont des groupes de cohomologie galoisienne (ou étale) .

- ${ }_{m} \operatorname{Br}(k)$ est la $m$-ième partie de torsion du groupe de Brauer de $k$ ( $m>0$ un entier).

- Si $F$ est un corps munie d'une valuation discrète $v$, l'anneau de valuation est noté par $\mathcal{O}_{v}$ et le corps résiduel, par $\kappa(v)$. L'extension maximale non ramifiée de $F$ est notée par $F_{n r}$. Si $x \in \mathcal{O}_{v}$, on note $\bar{x}$ son résidu dans $\kappa(v)$. On utilise cette notationci aussi pour d'autres objets munis de résidus naturels. Une valuation discrète est toujours supposée être non triviale (de rang 1 et de groupe des valeurs $\mathbb{Z}$ ).

- Soient $A$ une $k$-algèbre simple centrale et $F$ une extension de corps de $k$, alors $A_{F}:=A \otimes_{k} F$ est la $F$-algèbre simple centrale obtenue par extension de base.

Remerciements - L'auteur remercie vivement son directeur de thèse, Philippe Gille, pour soutenir cet article par ses idées et commentaires très utiles. Il remercie aussi la K.U.Leuven et l'École Normale Supérieure (Paris) pour le support financier et l'hospitalité qui ont réalisé tant de visites. L'auteur est aussi partiellement soutenu par «Fonds voor Wetenschappelijk Onderzoek Vlaanderen »(G.0318.06).

\section{Modules de cycles}

Dans cette section, on commence par décrire les groupes de cohomologie où l'invariant de Suslin a ses valeurs. Il sont des exemples privilégiés de modules de cycles. On donne une brève introduction à ce formalisme introduit par Rost [Ros1, §1,2], et puis on explique le lien avec les invariants cohomologiques d'un groupe algébrique découvert par Merkurjev [Mer1].

\subsection{Groupes de cohomologie}

Dans toute cette section, soient $F$ un corps de $\operatorname{car}(F)=p \geq 0$ et $m>0$ un entier inversible dans $F$.

(a) Définition - Soit $\mu_{m}^{\otimes i}$ la $i$-ième produit tensoriel de $\mu_{m}$ comme $\mathbb{Z} / m \mathbb{Z}$-module $(i \geq 0)$, alors on définit :

$$
H_{m}^{i}(F):=H^{i}\left(F, \mu_{m}^{\otimes i}(-1)\right) \quad \text { avec } \quad \mu_{m}^{\otimes i}(-1)=\operatorname{Hom}_{\Gamma_{F}}\left(\mu_{m}, \mu_{m}^{\otimes i}\right) .
$$

De plus, on pose $H_{m}^{i}(F)=0$ pour $i<0$. Bien évidemment, on a $\mu_{m}^{\otimes i+1}(-1)=\mu_{m}^{\otimes i}$ pour tout $i \geq 0$, et donc $H_{m}^{i+1}(F)=H^{i+1}\left(F, \mu_{m}^{\otimes i}\right) ! 1$ La suite exacte de Kummer

$$
1 \rightarrow \mu_{m}(F) \rightarrow F_{s}^{\times} \stackrel{m}{\rightarrow} F_{s}^{\times} \rightarrow 1
$$

implique l'interprétation cohomologique bien connue du sous-groupe de $m$-torsion du groupe de Brauer ${ }_{m} \operatorname{Br}(F) \cong H^{2}\left(F, \mu_{m}\right)$.

\footnotetext{
${ }^{1}$ On utilise le suscrit $i+1$ au lieu de $i$ juste par raisons de traditions et pour être conforme au cas sauvage où il semble un peu plus naturel d'utiliser ce suscrit.
} 
(b) Structure de $K_{n}(F)$-module - Considérons les $K$-groupes de Milnor ${ }^{2} K_{n}(F)$ pour un entier $n$. Rappelons que

$$
K_{n}(F)=\underbrace{F^{\times} \otimes_{\mathbb{Z}} \cdots \otimes_{\mathbb{Z}} F^{\times}}_{n \text { fois }} / J
$$

avec $J$ le sous-groupe engendré par des symboles de la forme $x_{1} \otimes \ldots \otimes x_{n}$ pour lequel $x_{i}+x_{j}=1$ pour $1 \leq i<j \leq n$. Les symboles primitifs sont notés abbrégés $\left\{x_{1}, \ldots, x_{n}\right\}$. La suite exacte de Kummer 2.1) implique au niveau de cohomologie $K_{1}(F) / m K_{1}(F)=$ $F^{\times} /\left(F^{\times}\right)^{m} \cong H^{1}\left(F, \mu_{m}\right)$. Utilisant le cup-produit, on obtient le symbole galoisien

$$
h_{m, F}^{n}: K_{n}(F) / m K_{n}(F) \rightarrow H^{n}\left(F, \mu_{m}^{\otimes n}\right)
$$

qui est un isomorphisme (conjecture de Bloch-Kato - Théorème de Voevodsky-Rost-Weibel [BK, Voe, Ros2, Wei] ). La structure de $K_{n}(F)$-module de $H_{m}^{i+1}(F)$ pour un entier $i \geq 0$ est défini par le cup produit avec le symbole galoisien :

$$
K_{n}(F) \times H_{m}^{i+1}(F) \rightarrow H_{m}^{n+i+1}(F):(a, b) \mapsto h_{m, F}^{n}(\bar{a}) \cup b .
$$

On note le produit scalaire par $a \cdot b:=h_{m, F}^{n}(\bar{a}) \cup b$ pour $a \in K_{n}(F)$, $\bar{a}$ sa classe dans $K_{n}(F) / m K_{n}(F)$ et $b \in H_{m}^{i+1}(F)$.

(c) Flèches de résidu - Si $v$ est une valuation discrète sur $F$ de corps résiduel $\kappa(v)$, on a une flèche de résidu pour tout entier $i \geq 0$,

$$
\partial_{v}^{i+1}: H_{m}^{i+1}(F) \rightarrow H_{m}^{i}(\kappa(v)),
$$

qui est bien expliquée par Serre [GMS, $\S 6,7]$. De plus, si $F$ est complet pour la valuation, elle a un scindage, parce que tout élément $\alpha$ de $H_{m}^{i+1}(F)$ s'écrit comme $\alpha_{i+1}+(\pi) \cup \alpha_{i}$ avec $\pi \in F$ un élément uniformisant, $(\pi)$ sa classe dans $F^{\times} /\left(F^{\times}\right)^{m}=H^{1}\left(F, \mu_{m}\right)$ et les $\alpha_{j} \in H_{m}^{j}(\kappa(v))$ se plongeant dans $H_{m}^{j}(F)$ [GMS, 7.11]. On a donc une suite exacte scindée intéressante :

$$
0 \rightarrow H_{m}^{i+1}(\kappa(v)) \rightarrow H_{m}^{i+1}(F) \stackrel{\partial_{v}^{i+1}}{\longrightarrow} H_{m}^{i}(\kappa(v)) \rightarrow 0 .
$$

(d) Version relative - Soit $A$ une $F$-algèbre simple centrale de $\operatorname{ind}_{F}(A)=n \in F^{\times}$. On considère sa classe de Brauer $[A] \in{ }_{n} \operatorname{Br}(F) \cong H^{2}\left(F, \mu_{n}\right)$. Utilisant le cup-produit de la cohomologie galoisienne, on définit (pour $i \geq 1$ )

$$
H_{n, A}^{i+1}(F):=H_{n}^{i+1}(F) /\left(H^{i-1}\left(F, \mu_{n}^{\otimes i-1}\right) \cup[A]\right) .
$$

Si $F$ est munie d'une valuation discrète $v$, on peut étendre les résidus de $H_{n}^{i+1}(F)$ à des résidus relatifs. On note $\bar{A}:=A \otimes_{\mathcal{O}_{v}} \kappa(v)$, la $\kappa(v)$-algèbre simple centrale résiduelle de $A$. La description en terme de cocycles explicites [GMS, §6] garantit bien que

$$
\partial_{v}^{i+1}\left(H^{i-1}\left(F, \mu_{n}^{\otimes i-1}\right) \cup[A]\right) \subset H^{i-2}\left(\kappa(v), \mu_{n}^{\otimes i-2}\right) \cup[\bar{A}] .
$$

\footnotetext{
${ }^{2}$ On utilise surtout des $K$-groupes de Milnor dans la suite. Donc, pour ne pas alourdir inutilement la notation, on oublie le suscrit $M$ de la notation usuelle $K_{n}^{M}(F)$ des $K$-groupes de Milnor, et on utilise la notation $K_{n}^{Q}$ pour les $K$-groupes de Quillen.
} 
Ça implique donc qu'on a un diagramme commutatif (pour $i \geq 2$ ) :

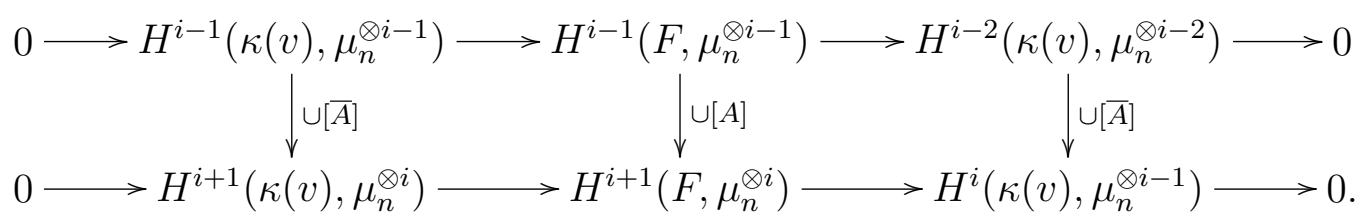

Le lemme du serpent permet donc de construire une suite exacte :

$$
0 \rightarrow H_{n, \bar{A}}^{i+1}(\kappa(v)) \rightarrow H_{n, A}^{i+1}(F) \stackrel{\partial_{v, A}^{i+1}}{\longrightarrow} H_{n, \bar{A}}^{i}(\kappa(v)) \rightarrow 0 .
$$

Puisque la suite exacte (2.4) est scindée, la suite exacte (2.5) l'est aussi. À noter que d'après la conjecture de Bloch-Kato et la structure de $K_{n}(F)$-module, on a une définition équivalente :

$$
H_{n, A}^{i+1}(F)=H^{i+1}\left(F, \mu_{n}^{\otimes i}\right) /\left(K_{i-1}(F) \cdot[A]\right) .
$$

\subsection{Définition}

Les propriétes communes des groupes de cohomologies $H_{n}^{*}(F)$ et des groupes de $K$ théorie de Milnor ont inspirés Rost à définir une structure formelle qui respecte les mêmes propriétés homologiques [Ros1, §1.2]. Nous rappelons en bref ce formalisme des modules de cycles.

(a) Définition d'un module de cycles - Soient $R$ un anneau de valuation discrète, $R$-corps la catégorie des $R$-corps (des $R$-algèbres qui sont des corps) et $\mathfrak{A} \mathfrak{b}$ la catégorie des groupes abéliens. Un module de cycles $M$ de base $R$ est un foncteur

$$
R-\mathfrak{c o r p s} \rightarrow \mathfrak{A} \mathfrak{b}
$$

munie d'une graduation $M=\left(M_{j}\right)_{j \geq 0}$ et des données D1-D4 $]^{3}(E, F$ des objets de $R$-corps et $\varphi$ un morphisme de $R$-corps)

D1 : Pour tout $\varphi: F \rightarrow E$, on a $\varphi_{*}: M(F) \rightarrow M(E)$ de degré 0 .

D2 : Pour tout $\varphi: F \rightarrow E$ fini, on a $\varphi^{*}: M(E) \rightarrow M(F)$ de degré 0 .

D3 : Pour tout $F$, le groupe $M(F)$ a une structure de $K_{n}(F)$-module tel que $K_{n}(F) \cdot M_{m}(F) \subset M_{n+m}(F)(n, m \geq 0$ des entiers).

D4 : Si $F$ est un $R$-corps de valuation discrète $v$, il existe un résidu $\partial_{v}: M(F) \rightarrow M(\kappa(v))$ de degré -1 .

Ces données doivent respecter des règles de compatibilité (R1a-R3e) et de géométrie (FD et C) - voir Appendice A et loc. cit. À noter que, pour obtenir ses buts, Rost lui-même met plus de restrictions sur la base $R$, mais il commente qu'il est permis de modérer les conditions (ibid., $§ 1$, p. 328).

\footnotetext{
${ }^{3} \mathrm{Si}$ on utilise $M_{j}$ pour $j<0$, dans une notation libre, il est 0.
} 
(b) La base et la coexistence de deux modules de cycles - Le cas classique est celui où la base est un corps. Dans ce texte, on utilise la terminologie des modules de cycles pour une base qui est un anneau $R$ de valuation discrète complet avec corps des fractions $K$ et corps résiduel $k$. Un module de cycles $M$ avec une telle base associe donc à toute extension de corps $L$ de $K$ un groupe gradué $M(L)$ et de même, à toute extension de corps $\bar{L}$ de $k$, un groupe gradué $M(\bar{L})$.

À noter que l'on peut bien restreindre un module de cycles de base $R$ en un module de cycles de base $K$ ou également de base $k$ en se restreignant aux extensions de corps de $K$ ou de $k$. Un module de cycles $M$ avec base $R$ est donc rien d'autre que la coexistence de deux modules de cycles avec un corps comme base (notés $\left.M\right|_{k}$ et $\left.M\right|_{K}$ ) avec un lien donné par D4. Dans la suite, on utilise les modules de cycles de base $R$ afin de faciliter la notation et de travailler dans un cadre plus général. Néanmoins, on pourrait reformuler les arguments, travailler avec deux modules de cycles et utiliser ces liens comme extérieurs aux modules de cycles mêmes : comme des données supplémentaires.

(c) Complexe de Gersten - Soient $F$ un $R$-corps, $X$ une $F$-variété et $M$ un module de cycles, alors l'existence des résidus et des règles de modules de cycles induisent un complexe de cycles de Gersten $C_{*}\left(X, M_{j}\right)$ [Ros1, $\left.\S 3.3\right](i, j \geq 0)$ :

$$
\ldots \rightarrow \oplus_{x \in X^{(i-1)}} M_{j-i+1}(F(x)) \stackrel{\partial^{i-1}}{\rightarrow} \oplus_{x \in X^{(i)}} M_{j-i}(F(x)) \stackrel{\partial^{i}}{\rightarrow} \oplus_{x \in X^{(i+1)}} M_{j-i-1}(F(x)) \rightarrow \ldots,
$$

où $X^{(i)}$ désigne l'ensemble des point de codimension $i$ dans $X$ et $F(x)$ est le corps résiduel de $x$, un point de codimension $i$. La flèche $\partial^{i}$ est bien la somme des résidus induits par les valuations associées à un point de codimension 1 de $X^{(i)}$. L'homologie de ce complexe au cran $i$ est notée $A^{i}\left(X, M_{j}\right)$.

(d) Exemples privilégiés - Lions cette section à la précédente. Soient $R$ un anneau de valuation discrète complet de corps des fractions $K$ et corps résiduel $k$ et pour une $K$ algèbre simple centrale $A$ de $\operatorname{ind}_{K}(A)=n$ tel que $n \in K^{\times}$et $n \in k^{\times}$. Alors, les foncteurs

$$
\begin{aligned}
\mathcal{H}_{m}^{*}:=\left(\mathcal{H}_{m}^{i}\right)_{i \geq 0}: R-\operatorname{corps} \rightarrow \mathfrak{A} \mathfrak{b} & : F \mapsto\left(H_{m}^{i}(F)\right)_{i \geq 0} \text { et } \\
\mathcal{H}_{n, \mathcal{A}}^{*}:=\left(\mathcal{H}_{n, \mathcal{A}}^{i}\right)_{i \geq 2}: R \text {-corps } \rightarrow \mathfrak{A b} & : F \mapsto\left(H_{n, A_{F}}^{i}(F)\right)_{i \geq 2}
\end{aligned}
$$

sont bien des modules de cycles où $A_{F}:=A \otimes_{R} F$. À noter que pour une extension $F$ de $K$, la $K$-algèbre $A_{F}$ est de nouveau simple centrale. On sait que $\operatorname{ind}_{k}(\bar{A})=n$ avec $\bar{A}=A \otimes_{R} k$ (voir démonstration du Corollaire 3.3). Alors pour tout $R$-corps $F, \operatorname{ind}\left(A_{F}\right) \mid n$ et $\left[A_{F}\right]$ se trouve donc bien dans ${ }_{n} \operatorname{Br}(F)$. La définition du deuxième module de cycles est donc consistante.

La vérification des règles du premier cas pour des $R$-corps d'égale caractéristique est décrite par Rost [Ros1, 1.11]. Le cas de caractéristique mixte suit de façon analogue. Le premier module de cycle induit la vérification du second puisque les données et règles se continuent au niveau relatif (voir aussi (2.5)).

Un autre exemple de modules de cycles est la $K$-théorie de Milnor. La donnée D1 est définie de façon évidente. Soit $E$ une extension finie de corps de $F$, alors la donnée D2 est induite par la norme $N_{E / F}$ appliquée aux générateurs [BT, Ch. I, §5]. La donnée D3 est définie par la structure multiplicative des $K$-groupes :

$$
K_{n}(F) \times K_{m}(F) \mapsto K_{n+m}(F):\left(\left\{x_{1}, \ldots, x_{n}\right\},\left\{y_{1}, \ldots, y_{m}\right\}\right) \mapsto\left(\left\{x_{1}, \ldots, x_{n}, y_{1}, \ldots y_{m}\right\}\right)
$$


Soit $F$ un corps de valuation discrète $v$, alors le résidu $K_{n}(F) \rightarrow K_{n-1}(\kappa(v))$-la donnée D4- est défini par

$$
\begin{aligned}
\left\{\pi, x_{2}, \ldots, x_{n}\right\} & \mapsto\left\{\bar{x}_{2}, \ldots, \bar{x}_{n}\right\} \\
\left\{x_{1}, x_{2}, \ldots, x_{n}\right\} & \mapsto 0
\end{aligned}
$$

avec $x_{1}, \ldots, x_{n} \in \mathcal{O}_{v}^{\times}$et $\pi$ une uniformisante de $F$ [Mil, Lem. 2.1].

\subsection{Lien avec les invariants}

Dans toute cette section, soient $k$ un corps et $M=\left(M_{j}\right)_{j \geq 0}$ un module de cycles de base $k$. Merkurjev a découvert un lien intéressant fécond entre les groupes $A^{i}\left(\mathbf{G}, M_{j}\right)$ et les invariants cohomologiques d'un $k$-groupe algébrique $\mathbf{G}$ dans $M$ de degré $j$. Expliquons-le, et commençons par rappeler la notion d'un invariant d'un groupe algébrique.

(a) Définition d'un invariant - Des invariants sont des objets fonctoriels, donc il faut des foncteurs. On considère un foncteur $\mathbf{G}: k-\mathfrak{c o r p s} \rightarrow \mathfrak{A b}$ (par exemple un groupe algébrique) et $M_{j}$ (pour $j \geq 0$ ) comme foncteur $k$-corps $\rightarrow \mathfrak{A} \mathfrak{b}$.

Un invariant $\rho$ de $\mathbf{G}$ dans $M$ de degré $j$ est une transformation naturelle des foncteurs $\mathbf{G} \rightarrow M_{j}$. En particulier, pour toute extension de corps $F$ de $k$, il consiste en un morphisme fonctoriel

$$
\rho_{F}: \mathbf{G}(F) \rightarrow M_{j}(F) .
$$

Tous les invariants de $\mathbf{G}$ dans $M$ de degré $j$ forment un groupe abélien, noté $\operatorname{Inv}^{j}(\mathbf{G}, M)$.

(b) Le lien de Merkurjev - Soit G un groupe algébrique, alors Merkurjev a construit un morphisme injectif

$$
\theta: \operatorname{Inv}^{j}(\mathbf{G}, M) \rightarrow A^{0}\left(\mathbf{G}, M_{j}\right): \rho \mapsto \rho_{K}(\xi),
$$

avec $K=k(\mathbf{G})$ et $\xi \in \mathbf{G}(K)$ le point générique de $\mathbf{G}$. Il démontre que l'image est le sousgroupe $A^{0}\left(\mathbf{G}, M_{j}\right)_{\text {mult }}$ contenant les éléments multiplicatifs de $A^{0}\left(\mathbf{G}, M_{j}\right)$ [Mer1, Lem. 2.1 et Thm. 2.3]. Ce sont les éléments $x \in A^{0}\left(\mathbf{G}, M_{j}\right)$ tels que

$$
p_{1}^{*}(x)+p_{2}^{*}(x)=m^{*}(x),
$$

où $p_{1}^{*}, p_{2}^{*}$ et $m^{*}$ sont les morphismes $A^{0}\left(\mathbf{G}, M_{j}\right) \rightarrow A^{0}\left(\mathbf{G} \times \mathbf{G}, M_{j}\right)$ induits par les deux projections $p_{1}, p_{2}: \mathbf{G} \times \mathbf{G} \rightarrow \mathbf{G}$ et la multiplication $m: \mathbf{G} \times \mathbf{G} \rightarrow \mathbf{G}$.

Il démontre aussi que $A^{0}\left(\mathbf{G}, M_{j}\right)_{\text {mult }} \subset \tilde{A}^{0}\left(\mathbf{G}, M_{j}\right)$, avec $\tilde{A}^{0}\left(\mathbf{G}, M_{j}\right)$ le sous-groupe réduit de $A^{0}\left(\mathbf{G}, M_{j}\right)$ (ibid., Lem. 1.9). Le sous-groupe réduit est le noyau du morphisme $u^{*}: A^{0}\left(\mathbf{G}, M_{j}\right) \rightarrow A^{0}\left(\mathbf{1}, M_{j}\right)$ qui est induit par le morphisme d'unité $u: \mathbf{1} \rightarrow \mathbf{G}$. Le morphisme $u^{*}$ induit même un scindage $A^{0}\left(\mathbf{G}, M_{j}\right) \cong \tilde{A}^{0}\left(\mathbf{G}, M_{j}\right) \oplus A^{0}\left(k, M_{j}\right)$.

(c) L'invariant de Suslin - Soient $k$ un corps et $A$ une $k$-algèbre simple centrale de norme réduite $\operatorname{Nrd}_{A / k}$, alors le groupe de Whitehead réduit $\operatorname{SK}_{1}(A)$ est isomorphe à $\mathrm{SL}_{1}(A) /\left[A^{\times}, A^{\times}\right]$. Considérant le foncteur (qui n'est pas un groupe algébrique)

$$
\mathbf{S K}_{1}(A): k \text {-corps } \rightarrow \mathfrak{A} \mathfrak{b}: F \mapsto \mathbf{S K}_{1}(A)(F)=\operatorname{SK}_{1}\left(A \otimes_{k} F\right)
$$


Suslin a introduit un invariant [Sus2, §3]

$$
\rho_{\mathrm{Sus}, A} \in \operatorname{Inv}^{4}\left(\mathbf{S K}_{1}(A), \mathcal{H}_{n, \mathcal{A}}^{*}\right) .
$$

Ici, $\mathcal{H}_{n, \mathcal{A}}^{*}=\left(\mathcal{H}_{n, A}^{j+1}\right)$ est un module de cycles de base $k$. Faisant de bonnes hypothèses sur $A$, on peut le voir comme un module de base d'un anneau $R$ complet de valuation discrète restreint à son corps de fractions ou à son corps résiduel selon \$2.2 (b) (voir aussi \$3.2 (b) .

Suslin démontre en plus que pour une extension de corps $F$ de $k$, les représentants de l'image de $\mathbf{S K}_{1}(A)(F)$ sont des élements du noyau de $H_{n}^{4}(F) \rightarrow H_{n}^{4}(F(X))$, où $X$ désigne la variété de Severi-Brauer associée à $A$ (loc. cit.).

\section{Le relèvement, le cas modéré}

Dans cette section, on utilise un relèvement de la caractéristique positive « modérée » à la caractéristique 0 afin de construire des invariants cohomologiques en caractéristique positive fondés sur l'existence des invariants en caractéristique 0. En plus, de relier les deux cas, on peut retrouver plus d'information sur la caractéristique positive moderée par la caractéristique 0 et vice versa.

\subsection{La stratégie}

Soient $k$ un corps et $A$ une $k$-algèbre simple centrale. Pour construire des invariants de $\mathbf{S K}_{1}(A)$, on voudrait utiliser l'isomorphisme de Merkurjev (2.7) qui nous permet d'obtenir des résultats en travaillant avec des complexes de Gersten. Malheureusement, cet isomorphisme ne vaut que pour des groupes algébriques, et $\mathbf{S K}_{1}(A)$ n'en est pas un. Dû à la projection $\mathbf{S L}_{1}(A)(F) \rightarrow \mathbf{S L}_{1}(A)(F) /\left[A_{F}^{\times}, A_{F}^{\times}\right] \cong \mathbf{S K}_{1}(A)(F)$ pour chaque extension de corps $F$ de $k$, on obtient quand-même une injection de groupes d'invariants.

Lemme 3.1. Soient $k$ un corps, $A$ une $k$-algèbre simple centrale et $M$ un module de cycles. La projection de $k$-foncteurs $\pi: \boldsymbol{S L}_{1}(A) \rightarrow \boldsymbol{S} \boldsymbol{K}_{1}(A)$ induit pour tout entier $j$ une injection

$$
\tilde{\pi}: \operatorname{Inv}^{j}\left(\boldsymbol{S K}_{1}(A), M\right) \hookrightarrow \operatorname{Inv}^{j}\left(\boldsymbol{S \boldsymbol { L } _ { 1 }}(A), M\right) .
$$

Nous utilisons donc des invariants de $\mathbf{S L}_{1}(A)$ qui nous permettent d'utiliser le résultat de Merkurjev. En plus, le travail que nous allons effectuer n'est pas fondé sur la définition de l'invariant de Suslin, mais sur son existence. Tout autre invariant analogue peut donc être relevé de la même façon.

Nous commençons par expliquer la stratégie générale du relèvement. Dans cette description, nous ne donnons pas d'arguments explicites et détaillés. Ceux-ci se trouvent dans les sections suivantes.

(i) Construire un invariant auxiliaire. Soit $k$ un corps de $\operatorname{car}(k)=p>0$ tel que $p$ ne divise pas $\operatorname{ind}_{k}(A)$. Il existe un anneau $R$ complet de valuation discrète $v$ tel que $k$ soit le corps résiduel et que le corps des fractions $K$ de $R$ est de caractéristique 0. Alors, $A$ se relève en une $R$-algèbre d'Azumaya $B$. De telle façon, $B_{K}:=B \otimes_{R} K$ est une $K$ algèbre simple centrale. Afin de construire un invariant dans $\operatorname{Inv}^{4}\left(\mathbf{S K}_{1}(A), \mathcal{H}_{n, A}^{*}\right)$, 
nous construisons un invariant auxiliaire $\rho^{\prime} \in \operatorname{Inv}^{3}\left(\mathbf{S K}_{1}(A), \mathcal{H}_{n, A}^{*}\right)$. Pour chaque extension de corps $k^{\prime}$ de $k$, il faut donc définir un morphisme

$$
\rho_{k^{\prime}}^{\prime}: \mathbf{S K}_{1}(A)\left(k^{\prime}\right) \rightarrow H_{n, A}^{3}\left(k^{\prime}\right) .
$$

Soit $K^{\prime}$ un corps complet de valuation discrète $w$ de corps résiduel $k^{\prime}$ tel que $K^{\prime}$ est une extension de $K$ et tel que $w$ prolonge $v$. Alors, on est donné un isomorphisme $\mathbf{S K}_{1}\left(B_{K}\right)\left(K^{\prime}\right) \rightarrow \mathbf{S K}_{1}(A)\left(k^{\prime}\right)$. À partir d'un invariant $\rho \in \operatorname{Inv}^{4}\left(\mathbf{S K}_{1}\left(B_{K}\right), \mathcal{H}_{n, B_{K}}^{*}\right)$, le résidu du module de cycles 2.5 donne un morphisme

$$
\rho_{k^{\prime}}^{\prime}: \mathbf{S K}_{1}(A)\left(k^{\prime}\right) \rightarrow H_{n, A}^{3}\left(k^{\prime}\right) .
$$

Celui-ci n'est pas nécessairement un invariant, puisque la fonctorialité en les extensions de corps n'est pas immédiatement obtenue. En effet, il y a plusieurs manières afin de trouver des corps de valuation $K^{\prime}$ comme ci-dessus. Pour résoudre ce problème, on utilise des anneaux de Cohen qui sont suffisamment canoniques.

(ii) En déduire l'invariant recherché. Puisque le résidu des modules de cycles se niche dans une suite exacte fonctorielle (2.5), on obtient un invariant de degré 4 dans $\operatorname{Inv}^{4}\left(\mathbf{S K}_{1}(A), \mathcal{H}_{n, A}^{*}\right)$ dès que l'invariant $\rho^{\prime}$ est trivial. D'après le Lemme 3.1, pour démontrer la trivialité, il suffit de démontrer la trivialité de l'invariant $\tilde{\pi}\left(\rho^{\prime}\right)$ de $\mathbf{S L}_{1}(A)$. Comme annoncé, on utilise le morphisme $\theta$ de Merkurjev (2.7) pour ce but. On démontre que $\theta\left(\tilde{\pi}\left(\rho^{\prime}\right)\right)=0$ en travaillant sur la définition des groupes $\tilde{A}^{0}$ et utilisant des résultats connus.

\subsection{Objets de base}

Avant de relever des invariants, on doit être capable de relever les objets de base d'une façon adaptée. Nous expliquons donc en bref le relèvement des corps et des algèbres simples centrales.

(a) Algèbres simples centrales - Soient $k$ un corps et $A$ une $k$-algèbre simple centrale. Soit $R$ un anneau complet de valuation discrète $v$ tel que $k$ soit le corps résiduel et tel que $K=\operatorname{Frac}(R)$ est de caractéristique 0 (par exemple, un anneau de Cohen $R$ avec son corps de fractions - voir (b) .

Soient $P(R)$, respectivement $P(k)$, l'ensemble des classes d'isomorphismes des $R$ algèbres d'Azumaya, respectivement des $k$-algèbres simples centrales. Alors, l'application résiduelle $P(R) \rightarrow P(k)$ qui associe à la classe d'une $R$-algèbre d'Azumaya $B$ la classe de $B \otimes_{R} k$, est bijective Gro2, Thm. 6.1]. Alors, il existe une $R$-algèbre d'Azumaya relevée (i.e. tel que $B \otimes_{R} k \cong A$ ) de $A$ de même indice et degré, appelons-la $B$. Alors, $B_{K}:=B \otimes_{R} K$ est une $K$-algèbre simple centrale. La bijection $P(R) \rightarrow P(k)$ induit un isomorphisme $\operatorname{Br}(R) \cong \operatorname{Br}(k)$, et de plus il y a une injection $\operatorname{Br}(R) \rightarrow \operatorname{Br}(K)$ [AG, Thm. 7.2]. Donc en somme, on a une injection $\operatorname{Br}(k) \rightarrow \operatorname{Br}(K)$.

Heureusement, $\mathbf{S K}_{1}(A)(k)$ et $\mathbf{S K}_{1}\left(B_{K}\right)(K)$ sont isomorphes. Ce résultat est essentiellement dû à Platonov pour des algèbres à division. Pour une $K$-algèbre à division $D$, la valuation $v$ s'étend à une valuation $w=\frac{1}{m} v \circ \operatorname{Nrd}_{D / K}$ sur $D$ avec $\operatorname{Nrd}_{D / K}$ la norme réduite de $D$ et $m>0$ le générateur de $v \circ \operatorname{Nrd}_{D / K}(D) \subset \mathbb{Z}$ [Ser1, Ch. XII, §2]. Soit $\mathcal{O}_{D}$ l'anneau de valuation de $w$ avec idéal maximal $\mathcal{P}_{D}$, alors on note $\bar{D}=\mathcal{O}_{D} / \mathcal{P}_{D}$, la $k$-algèbre à division résiduelle - voir aussi [Wad, §2]. L'application résiduelle $\mathcal{O}_{D} \rightarrow \bar{D}$ se restreint alors 
à un morphisme résiduel $\mathbf{S L}_{1}(D)(K) \rightarrow \mathbf{S L}_{1}(\bar{D})(k)$, et Platonov a démontré la propriété de rigidité suivante.

Théorème 3.2 ([Pla, Prop. 3.4, Thm. 3.12, Cor. 3.13]). Soient $K$ un corps complet de valuation discrète $v$ avec $k$ son corps résiduel et $D$ une $K$-algèbre à division. Le morphisme résiduel

$$
\boldsymbol{S L}_{1}(D)(K) \rightarrow \boldsymbol{S} \boldsymbol{L}_{1}(\bar{D})(k)
$$

est surjectif de noyau contenu dans $\left[D^{\times}, D^{\times}\right]$. Il factorise donc à travers $\boldsymbol{S K}_{1}(D)$, et cette factorisation induit un isomorphisme

$$
\boldsymbol{S K}_{1}(D)(K) \cong \boldsymbol{S} \boldsymbol{K}_{1}(\bar{D})(k) .
$$

Le but est d'en déduire l'isomorphisme entre $\mathbf{S K}_{1}(A)(k)$ et $\mathbf{S K}_{1}\left(B_{K}\right)(K)$. Bien évidemment, on utilise les théorèmes consacrés de Wedderburn et de Morita GS, Thm. 2.1.3, Lem. 2.8.6].

Corollaire 3.3. Soient $A, B, k, R$ et $K$ comme dessus, alors

$$
\boldsymbol{S K}_{1}(A)(k) \cong \boldsymbol{S} \boldsymbol{K}_{1}\left(B_{K}\right)(K)
$$

Démonstration. Selon le théorème de Wedderburn, $B_{K}=M_{m}(D)$ pour une $K$-algèbre à division $D$ et $m>0$. Dû à l'injectivité de $\operatorname{Br}(R) \rightarrow \operatorname{Br}(K)$, on sait que $M_{m}\left(\mathcal{O}_{D}\right)$ est Brauer-équivalent à $B$. Donc de nouveau dû à Wedderburn, $A=M_{m}(\bar{D})$. Alors, le Théorème 3.2 et le théorème de Morita garantissent que

$$
\mathbf{S K}_{1}\left(B_{K}\right)(K) \cong \mathbf{S K}_{1}(D)(K) \cong \mathbf{S K}_{1}(\bar{D})(k) \cong \mathbf{S K}_{1}(A)(k) .
$$

Remarque 3.4 - Il faut noter aussi que cet isomorphisme est fonctoriel dans le sens suivant. Soit $K^{\prime}$ une extension de corps de $K$ qui est également complet et de valuation discrète $v^{\prime}$ tel que $v^{\prime}$ prolonge $v$ et soit $k^{\prime}$ le corps résiduel de $K^{\prime}$. Alors, l'isomorphisme commute avec l'extension de base de $K$ à $K^{\prime}$ et de $k$ à $k^{\prime}$. On n'a quand même pas d'équivalence de foncteurs, puisque il n'y a pas de bijection entre les extensions de $k$ et celles de $K$.

(b) Anneaux de Cohen - Les anneaux de Cohen - parfois aussi appelés des p-anneauxfournissent une méthode assez canonique de relever des corps de caractéristique positive à des anneaux de caractéristique 0 . Nous commençons par la définition d'un anneau de Cohen.

Définition 3.5. Un anneau de Cohen est un anneau complet de valuation discrète dont le corps résiduel est de caractéristique $p>0$, et dont l'idéal maximal est engendré par $p$. Pour un premier $p$ fixé, on parle des p-anneaux de Cohen.

Schoeller donne une construction explicite de ces anneaux [Sch, §3]. Ils sont des sousanneaux des anneaux de Witt. Dans le cas où le corps résiduel est parfait, ils sont exactement les anneaux de Witt. En général, l'anneau de Cohen contient l'anneau de Witt de son sous-corps parfait maximal. À noter aussi que les anneaux de Cohen sont de caractéristique 0 . Nous rappelons le résultat fondamental sur ces anneaux. 
Théorème 3.6 ([Coh], voir aussi [Gro1, Thm. 19.8.6]).

(i) Soient $W$ un anneau de Cohen, $C$ un anneau local noethérien complet et I un idéal de $C$ distinct de $C$. Alors tout homomorphisme local $u: W \rightarrow C / I$ se factorise en $W \stackrel{v}{\rightarrow} C \rightarrow C / I$, où $v$ est un homomorphisme local.

(ii) Soit $k$ un corps de caractéristique $p>0$. Il existe un anneau de Cohen $W$ dont le corps résiduel est isomorphe à $k$. Si $W^{\prime}$ est un second anneau de Cohen, $k^{\prime}$ son corps résiduel, tout isomorphisme $u: k \rightarrow k^{\prime}$ provient par passage au quotient d'un isomorphisme $v: W \rightarrow W^{\prime}$.

Remarque 3.7 - Remarquons que la propriété (i) induit que les anneaux de Cohen sont des objets initiaux dans la catégorie des anneaux locaux avec corps résiduel fixe. Ce théorème semble donc suggérer qu'il existe une propriété universelle des anneaux de Cohen. Malheureusement, les morphismes induits ne sont pas uniques en général. Il ne le sont que si $k$ est parfait (avec $k$ le corps résiduel de $W$ ) [Gro1, Rem. 21.5.3]. Donc par manque d'unicité, on appelle cette propriété universelle amputée une propriété verselle, comme le fait Serre [GMS, §5]. Heureusement cet handicap se guérit au niveau de la cohomologie.

Corollaire 3.8. Soient $W, W^{\prime}$ des anneaux de Cohen tel que le corps résiduel $k^{\prime}$ de $W^{\prime}$ est une extension de $k$, le corps résiduel de $W$. Notons $u: k \rightarrow k^{\prime}$ l'inclusion. Le Théorème 3.6 (i) fournit un homomorphisme local $v: W \rightarrow W^{\prime}$. Soient $A$ une k-algèbre simple centrale et $B$ la $W$-algèbre d'Azumaya relevée. Soient $K=\operatorname{Frac}(W)$ et $K^{\prime}=\operatorname{Frac}\left(W^{\prime}\right)$, alors $v$ définit pour tous entiers $i, n \geq 0$ un homomorphisme des suites exactes scindées

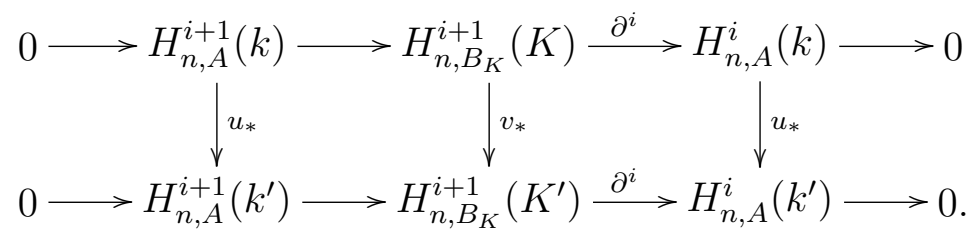

De plus, $v_{*}$ ne dépend pas du choix de $v$. Si $k=k^{\prime}$, on a un isomorphisme canonique $H_{n, B_{K}}^{i+1}(K) \cong H_{n, B_{K}}^{i+1}\left(K^{\prime}\right)$.

Démonstration. Soient $k, k^{\prime}$ de caractéristique $p$, l'homomorphisme local $v$ envoie l'uniformisante $p \in W$ sur $p \in W^{\prime}$. Donc, le diagramme et l'indépendance du choix de $v$ suivent directement du scindage de la suite (2.5) par le cup-produit avec la classe de $p$. Si $u$ est un isomorphisme, $v$ l'est aussi par le Théorème 3.6 (ii), et on retrouve un isomorphisme de suites exactes.

Les anneaux de Cohen fournissent donc au niveau de ces groupes de cohomologie une méthode canonique pour passer de la caractéristique $p$ à la caractéristique 0 , et vice versa. Pour faciliter la notation, introduisons une notion de triplets.

Définition 3.9. Soit $F$ un corps de valuation discrète $v$, alors on dit que $\left(F, \mathcal{O}_{v}, \kappa(v)\right)$ est un triplet de valuation. Un triplet de valuation $(K, R, k)$ où $R$ est un p-anneau de Cohen (pour un premier $p>0$ ) est appelé un p-triplet de Cohen. Une extension de Cohen (finie, resp. séparable, resp. galoisienne) $\left(K^{\prime}, R^{\prime}, k^{\prime}\right)$ de $(K, R, k)$ est la donnée d'un p-triplet de Cohen tel que $k^{\prime}$ est une extension (finie, resp. séparable, resp. galoisienne) de $k$. 
Remarque 3.10 - Si cela ne prête pas à confusion, on parle librement d'un triplet de Cohen sans mentionner la caractéristique. Étant donné un corps $k$ de $\operatorname{car}(k)=p>0$, le Théorème 3.6 fournit un triplet de Cohen $(K, R, k)$ (non unique) associé à $k$. De plus, si $\left(K^{\prime}, R^{\prime}, k^{\prime}\right)$ est une extension de Cohen (finie, resp. séparable, resp. galoisienne) de $(K, R, k)$, le Théorème 3.6 induit que $K^{\prime}$ est une extension (finie, resp. non-ramifiée, resp. galoisienne) de $K$ - voir aussi [Ser1, $§ I I I .5]$. Si $(K, R, k)$ est un triplet de Cohen, $F$ un $R$-corps et $\left(F, \mathcal{O}_{v}, \kappa(v)\right)$ un triplet de valuation, on dit que $\left(F, \mathcal{O}_{v}, \kappa(v)\right)$ est un triplet de valuation sur $R$. Dans ce cas, $\kappa(v)$ est aussi un $R$-corps et il y a trois possibilités : ou bien $F$ et $\kappa(v)$ sont des extensions de $K$, ou bien ils sont toutes les deux des extensions de $k$, ou bien $F$ est une extension de $K$ et $\kappa(v)$ est une extension de $k$.

\subsection{Le relèvement}

On a maintenant fait les préparations pour relever l'invariant de Suslin en caractéristique modérée.

Théorème 3.11. Soient $k$ un corps de $\operatorname{car}(k)=p>0$ et $A$ une $k$-algèbre simple centrale de ind $d_{k}(A)=n$ premier à $p$. Soit $(K, R, k)$ un triplet de Cohen associé à $k, B$ la $R$-algèbre d'Azumaya relevée et $\rho^{\prime} \in \operatorname{Inv}^{4}\left(\boldsymbol{S} \boldsymbol{K}_{1}\left(B_{K}\right), \mathcal{H}_{n, \mathcal{B}_{K}}^{*}\right)$. Alors, il existe un unique $\rho \in \operatorname{Inv}^{4}\left(\boldsymbol{S K}_{1}(A), \mathcal{H}_{n, \mathcal{A}}^{*}\right)$, que l'on appelle l'invariant spécialisé de $\rho^{\prime}$, tel que pour toute extension de Cohen $\left(K^{\prime}, R^{\prime}, k^{\prime}\right)$ de $(K, R, k)$ le diagramme suivant commute:

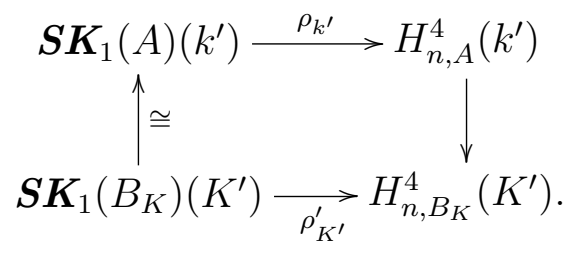

Remarque 3.12 - Les modules de cycles $\mathcal{H}_{n, \mathcal{B}_{K}}^{*}:=\left(H_{n, B_{K}}^{j}\right)_{j \geq 2}$ de base $K$ et $\mathcal{H}_{n, \mathcal{A}}^{*}:=$ $\left(H_{n, A}^{j}\right)_{j \geq 2}$ de base $k$ sont les modules de cycles évidents. Il sont les modules de cycles restreints du module de cycles $\mathcal{H}_{n, \mathcal{B}_{K}}^{*}$ de base $R$ respectivement à $K$ et à $k$ (selon $\$ 2.2$ (b). Le morphisme $H_{n, A}^{4}\left(k^{\prime}\right) \rightarrow H_{n, B_{K}}^{4}\left(K^{\prime}\right)$ est donc l'injection de la suite exacte (2.5).

Commençons par le deuxième pas de la stratégie générale expliquée dans 3.1. On utilise la proposition suivante qui est une donnée importante.

Proposition 3.13 (Merkurjev [Mer1, Lem. 4.8 et Prop. 4.9]). Soient $k$ un corps et $\boldsymbol{G}$ un $k$-groupe semi-simple simplement connexe, alors $\tilde{A}^{0}\left(\boldsymbol{G}, \mathcal{H}_{n}^{3}\right)=0$ pour tout $n \in k^{\times}$. En particulier (d'après $\$ 2.3(b), \operatorname{Inv}^{3}\left(\boldsymbol{G}, \mathcal{H}_{n}^{*}\right)=0$.

Nous nous permettons d'en déduire un résultat auxiliaire à base de quelques arguments homologiques.

Corollaire 3.14. Soient $k$ un corps, $\boldsymbol{G}$ un $k$-groupe semi-simple simplement connexe, $A$ une $k$-algèbre simple centrale tel que $\operatorname{ind}_{k}(A)=n \in k^{\times}$, alors $\operatorname{Inv}^{3}\left(\boldsymbol{G}, \mathcal{H}_{n, \mathcal{A}}^{*}\right)=0$.

Démonstration. Selon $2.3(\mathrm{~b})$, il suffit de démontrer la trivialité de $\tilde{A}^{0}\left(\mathbf{G}, \mathcal{H}_{n, \mathcal{A}}^{3}\right)$. Tout 
d'abord on considère le diagramme commutatif

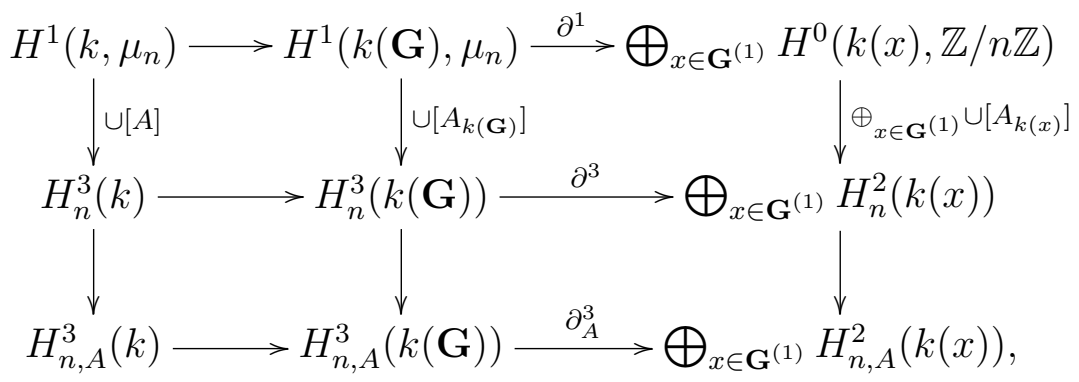

dont les lignes sont des complexes de chaînes, et dont la ligne centrale est exacte vu la Proposition 3.13 et l'isomorphisme (2.7). Il suffit de démontrer l'exactitude de la ligne d'en bas. Le théorie de Kummer et les propriétés des résidus [GMS, Rem. 6.2] indiquent que $\partial^{1}$, qui est une somme des résidus, est en réalité le morphisme diviseur :

$$
k(\mathbf{G})^{\times} /\left(k(\mathbf{G})^{\times}\right)^{n} \rightarrow \bigoplus_{x \in \mathbf{G}^{(1)}} \mathbb{Z} / n \mathbb{Z}=\operatorname{Div}(\mathbf{G}) / n \operatorname{Div}(\mathbf{G}): \bar{f} \mapsto \overline{\operatorname{div}(f)} .
$$

Il est surjectif puisque $\operatorname{Pic}(\mathbf{G})=0$ [San, Lem. 6.9]. Se basant sur la surjectivité de $\partial^{1}$, on démontre alors l'exactitude du complexe en bas avec une chasse au diagramme.

Démonstration du Théorème 3.11. Soit $\rho^{\prime} \in \operatorname{Inv}^{4}\left(\mathbf{S K}_{1}\left(B_{K}\right), \mathcal{H}_{n, \mathcal{B}_{K}}^{*}\right)$. Afin de construire $\rho \in \operatorname{Inv}^{4}\left(\mathbf{S K}_{1}(A), \mathcal{H}_{n, \mathcal{A}}^{*}\right)$, il faut commencer par définir $\rho_{k^{\prime}}: \mathbf{S K}_{1}(A)\left(k^{\prime}\right) \rightarrow H_{n, A}^{4}\left(k^{\prime}\right)$ pour toute extension de corps $k^{\prime}$ de $k$, et puis prouver la fonctorialité en le corps. Soit donc $\left(K^{\prime}, R^{\prime}, k^{\prime}\right)$ une extension de Cohen associée à $k^{\prime}$. On a donc $\rho_{K^{\prime}}^{\prime}: \mathbf{S K}_{1}\left(B_{K}\right)\left(K^{\prime}\right) \rightarrow$ $H_{n, B_{K}}^{4}\left(K^{\prime}\right)$. Soit $\pi$ l'isomorphisme $\mathbf{S K}_{1}\left(B_{K^{\prime}}\right)\left(K^{\prime}\right) \cong \mathbf{S K}_{1}(A)(k)$, alors on définit

$$
\tilde{\rho}_{k^{\prime}}:=\partial^{4} \circ \rho_{K^{\prime}} \circ \pi^{-1}: \mathbf{S K}_{1}(A)\left(k^{\prime}\right) \rightarrow H_{n, A}^{3}\left(k^{\prime}\right) .
$$

À noter aussi que ce procédé ne dépend pas du choix de l'extension de Cohen. Car si $\left(K^{\prime \prime}, R^{\prime \prime}, k^{\prime}\right)$ est une autre extension de Cohen associée à $k^{\prime}$, le Corollaire 3.8 garantit un isomorphisme des suites exactes scindées de type (2.5) avec l'identité au facteurs $H_{n, A}^{4}\left(k^{\prime}\right)$ et $H_{n, A}^{3}\left(k^{\prime}\right)$. De plus $\rho_{K^{\prime}}^{\prime}$ et $\pi$ sont fonctoriels pour de telles extensions de corps, donc ce procédé construit bien un invariant $\tilde{\rho} \in \operatorname{Inv}^{3}\left(\mathbf{S K}_{1}(A), \mathcal{H}_{n, \mathcal{A}}^{*}\right)$.

Le Corollaire 3.14 et la Propriété 3.1 disent alors que $\tilde{\rho}=0$. C'est-à-dire si $a \in$ $\mathbf{S K}_{1}(A)\left(k^{\prime}\right)$, on sait que $\rho_{k^{\prime}}^{\prime}(a)$ provient d'un élément unique de $H_{n, A}^{4}\left(k^{\prime}\right)$ (par la suite exacte (2.5)). Donc on a un morphisme $\rho_{k^{\prime}}: \mathbf{S K}_{1}(A)\left(k^{\prime}\right) \rightarrow H_{n, A}^{4}\left(k^{\prime}\right)$. De nouveau puisque, la suite (2.5) est fonctorielle, et le choix de l'anneau de Cohen n'a pas d'influence sur la définition, ceci définit un invariant $\rho \in \operatorname{Inv}^{4}\left(\mathbf{S K}_{1}(A), \mathcal{H}_{n, \mathcal{A}}^{*}\right)$.

Le diagramme commutatif (3.1) suit de la construction, et l'unicité suit immédiatement de l'injectivité de $H_{n, A}^{4}\left(k^{\prime}\right) \rightarrow H_{n, B_{K}}^{4}\left(K^{\prime}\right)$.

Remarque 3.15 - Pour un corps $k$ de $\operatorname{car}(k)=p>0$ et $A$ une $k$-algèbre simple centrale de $\operatorname{ind}_{k}(A) \in k^{\times}$, Suslin a déjà defini un invariant $\rho_{\mathrm{Sus}, A}$. Néanmoins, si $(K, R, k)$ est un $p$-triplet de Cohen, $B$ la $R$-algèbre d'Azumaya relevée et $\rho_{\mathrm{Sus}, B_{K}}$ l'invariant de Suslin de $B_{K}$, l'invariant spécialisé de $B_{K}$ est quand même le même invariant que $\rho_{\text {Sus, } A}$. En effet, on peut vérifier que l'invariant de Suslin satisfait cette propriété de relèvement (i.e. il existe un diagramme comme (3.1) avec $\rho=\rho_{\mathrm{Sus}, A}$ et $\left.\rho^{\prime}=\rho_{\mathrm{Sus}, B_{K}}\right)$. 


\section{Le relèvement, le cas sauvage}

Soient $k$ un corps de caractéristique $p>0$ et $A$ une $k$-algèbre simple centrale d'indice éventuellement divisible par $p$. On se trouve maintenant dans un monde inconnu, puisque le module de cycles $\mathcal{H}_{n, \mathcal{A}}^{*}$ n'est plus adapté à nos buts. En effet, parce que $\mu_{p^{n}}\left(k_{s}\right)$ est trivial, les groupes de cohomologie galoisienne $H^{j+1}\left(k, \mu_{p^{n}}^{\otimes j}\right)$ sont triviaux, et la suite exacte de Kummer (2.1) ne donne plus d'isomorphisme de $H^{2}\left(k, \mu_{p^{n}}\right)$ avec ${ }_{p^{n}} \operatorname{Br}(k)$.

Dans cette section, on décrit de nouveaux groupes de cohomologie (introduits par Kato [Kat1]) qui fournissent aussi un isomorphisme avec ${ }_{p^{n}} \operatorname{Br}(k)$ (dont on a besoin afin de pouvoir définir des modules de cycles relatifs comme dans $2.1(\mathrm{~d})$ ) . Ceci donne assez d'ingrédients pour effectuer le relèvement. Dans une première étape, nous effectuons le travail pour une algèbre simple centrale d'indice $p^{n}$. Le relèvement pour le cas général peut en être déduit utilisant la décomposition d'une algèbre à division en parties premières $(\S 4.3)$.

\subsection{Groupes de cohomologie}

On utilise des groupes de cohomologie des différentielles logarithmiques de Kato (ibid.). Ils sont pourvus d'une suite exacte analogue à la suite (2.5) dont on a besoin afin d'établir le relèvement. Soient donc dans toute cette section $(K, R, k)$ un $p$-triplet de Cohen et $F$ un $R$-corps. Rappelons la notion de différentielles logarithmiques et la définition, ainsi que quelques propriétes des groupes $H_{p^{n}}^{q+1}(k)$ (pour des entiers $n, q \geq 0$ )

(a) Différentielles logarithmiques - La définition de $H_{p^{n}}^{q+1}(k)$ est le plus claire pour $n=1$ et explique la terminologie. Soient $\Omega_{k}^{q}:=\bigwedge \Omega_{k / \mathbb{Z}}^{1}$ et $d: \Omega_{k}^{q-1} \rightarrow \Omega_{k}^{q}$ la dérivation extérieure usuelle (pour $q=0$, on pose $d=0$ ). Alors, $H_{p}^{q+1}(k)$ est défini comme le conoyau du morphisme de Cartier

$F-1: \Omega_{k}^{q} \rightarrow \Omega_{k}^{q} / d \Omega_{k}^{q-1}, \quad$ defini par $\quad x \frac{d y_{1}}{y_{1}} \wedge \ldots \wedge \frac{d y_{q}}{y_{q}} \mapsto\left(x^{p}-x\right) \frac{d y_{1}}{y_{1}} \wedge \ldots \wedge \frac{d y_{q}}{y_{q}} \bmod d \Omega_{k}^{q-1}$, avec $x \in k, y_{1}, \ldots, y_{q} \in k^{\times}$et $F(x)=x^{p}$ [Car, Ch. 2, §6]. Le noyau est noté traditionellement $\nu_{1}(q)_{k}$.

(b) Généralisation - On peut généraliser la définition de $H_{p}^{q+1}(k)$ à une définition de $H_{p^{n}}^{q+1}(k)$. Soit

$$
D_{p^{n}}^{q}(k)=W_{n}(k) \otimes \underbrace{k^{\times} \otimes \ldots \otimes k^{\times}}_{q \text { fois }},
$$

avec $W_{n}(k)$ le groupe des $p$-vecteurs de Witt de longueur $n$ sur $k$. Soit $J_{q}^{\prime}(k)$ le sous-groupe de $D_{p^{n}}^{q}(k)$ engendré par tous les éléments de la forme

(i) $w \otimes b_{1} \otimes \ldots \otimes b_{q}$, satisfiant $b_{i}=b_{j}$ pour $1 \leq i<j \leq q$.

\footnotetext{
${ }^{4}$ L'indice $q+1$ est de nouveau dû à la tradition facilitant la notation de quelques résultats.
} 
Le groupe $C_{p^{n}}^{q}(k)=D_{p^{n}}^{q}(k) / J_{q}^{\prime}(k)$ est alors muni d'une dérivation $d: C_{p^{n}}^{q-1}(k) \rightarrow C_{p^{n}}^{q}(k)$ : pour $a \in k, b_{2}, \ldots, b_{q} \in k^{\times}$est $q>0$ définie par

$$
(0, \ldots, 0, a, 0, \ldots, 0) \otimes b_{2} \otimes \otimes \ldots b_{q} \mapsto(0, \ldots, 0, a, 0, \ldots, 0) \otimes a \otimes b_{2} \otimes \ldots \otimes b_{q} .
$$

Pour $q=0$, on pose de nouveau $d=0$. Le groupe de cohomologie $H_{p^{n}}^{q+1}(k)$ est alors défini comme le conoyau du morphisme de Cartier

$$
\begin{aligned}
F-1: C_{p^{n}}^{q}(k) & \rightarrow C_{p^{n}}^{q}(k) / d C_{p^{n}}^{q-1}(k), \quad \text { défini par } \\
w \otimes b_{1} \otimes \ldots \otimes b_{q} & \mapsto\left(w^{(p)}-w\right) \otimes b_{1} \otimes \ldots \otimes b_{q} .
\end{aligned}
$$

Ici, $F(w)=w^{(p)}=\left(a_{1}^{p}, \ldots, a_{n}^{p}\right)$ si $w=\left(a_{1}, \ldots, a_{n}\right)$. On note $\nu_{n}(q)_{k}$ le noyau du morphisme de Cartier. Alors, $H_{p^{n}}^{q+1}(k) \cong D_{p^{n}}^{q}(k) / J_{q}(k)$ où $J_{q}(k)$ [Kat1, Proof of Prop. 2] est le sousgroupe de $D_{p^{n}}^{q}(k)$ engendré par les éléments de la forme (i) et

(ii) $(0, \ldots, 0, a, 0, \ldots, 0) \otimes a \otimes b_{2} \otimes \ldots \otimes b_{q}$,

(iii) $\left(w^{(p)}-w\right) \otimes b_{1} \otimes \ldots \otimes b_{q}$.

De plus, on pose $H_{p^{n}}^{q+1}(k)=0$ pour $q<0$. Cette construction est donc bien une généralisation de la définition pour $n=1$ en termes de différentielles logarithmiques par l'identification

$$
x \frac{d y_{1}}{y_{1}} \wedge \ldots \wedge \frac{d y_{q}}{y_{q}} \leftrightarrow x \otimes y_{1} \otimes \ldots \otimes y_{q}
$$

avec $x \in k$ et $y_{i} \in k^{\times}$. À noter que l'antisymétrie vaut pour cette généralisation, puisque $w \otimes b_{1} b_{2} \otimes b_{1} b_{2} \otimes \ldots b_{q}=0\left(w \in W_{n}(k), b_{i} \in k^{\times}\right)$.

Soit dlog $: k_{s}^{\times} \rightarrow \nu_{n}(1)_{k_{s}}: a \mapsto 1 \otimes a$. La suite exacte longue associée à la suite exacte

$$
1 \longrightarrow k_{s}^{\times} \stackrel{p^{n}}{\longrightarrow} k_{s}^{\times} \stackrel{\operatorname{dlog}}{\longrightarrow} \nu_{n}(1)_{k_{s}} \longrightarrow 1
$$

des $\Gamma_{k}$-modules induit un isomorphisme sur la partie de $p^{n}$-torsion du groupe de Brauer : $H^{1}\left(k, \nu_{n}(1)_{k_{s}}\right) \cong{ }_{p^{n}} \operatorname{Br}(k)$. L'exactitude de (4.1) suit d'un calcul avec des vecteurs de Witt et des produits tensoriels [Car, Ch. 2, Prop. 8]. De plus, on a une suite exacte :

$$
0 \longrightarrow \nu_{n}(q)_{k_{s}} \longrightarrow C_{p^{n}}^{q}\left(k_{s}\right) \stackrel{F-1}{\longrightarrow} C_{p^{n}}^{q}\left(k_{s}\right) / d C_{p^{n}}^{q-1}\left(k_{s}\right) \longrightarrow 0 .
$$

La surjectivité de $F-1$ suit du Théorème 4.1 qui démontre que $H_{p^{n}}^{q+1}\left(k_{s}\right)=0$ pour chaque $q \geq 0$ et $n>0$. Puisque $C_{p^{n}}^{q}\left(k_{s}\right)$ est un $k_{s^{-}}$espace vectoriel tel que $C_{p^{n}}^{q}\left(k_{s}\right)^{\Gamma}=C_{p^{n}}^{q}(k)$, on en déduit donc par le théorème de Hilbert 90 additif l'isomorphisme

$$
H^{1}\left(k, \nu_{n}(q)_{k_{s}}\right) \cong H_{p^{n}}^{q+1}(k)
$$

Comme dans le cas modéré on a donc

$$
H_{p^{n}}^{2}(k) \cong{ }_{p^{n}} \operatorname{Br}(k)
$$


(c) Suite exacte de Kato - Afin de pouvoir établir le même canevas que dans le cas modéré, il faut qu'on ait un module de cycles et on aimerait aussi avoir une suite exacte comme (2.5). Malheureusement, la théorie est plus complexe, mais on a quand même le résultat suivant.

Théorème 4.1 (Kato [Kat1], Izhboldin [Izh]). Soit $F$ un $R$-corps complet de valuation discrète $v$ avec triplet de valuation $\left(F, \mathcal{O}_{v}, \kappa(v)\right)$. Soient $F_{n r}$ l'extension maximale nonramifiée de $F$ et

$$
H_{p^{n}, n r}^{q+1}(F):=\operatorname{ker}\left(H_{p^{n}}^{q+1}(F) \rightarrow H_{p^{n}}^{q+1}\left(F_{n r}\right)\right)
$$

Alors, on a une suite exacte scindée

$$
0 \rightarrow H_{p^{n}}^{q+1}(\kappa(v)) \rightarrow H_{p^{n}, n r}^{q+1}(F) \rightarrow H_{p^{n}}^{q}(\kappa(v)) \rightarrow 0 .
$$

Remarque 4.2 - Expliquons le scindage et les morphismes en jeu. Selon les caractéristiques de $F$ et $\kappa(v)$, on discute trois situations.

- Dans le cas d'inégale caractéristique $(\operatorname{car}(F)=0$ et $\operatorname{car}(\kappa(v))=p)$, le scindage est obtenu par des morphismes dûs à Kato [Kat1, Proof of Prop. 2]. D'une part, on a une inclusion $i^{*}: H_{p^{n}}^{q+1}(\kappa(v)) \rightarrow H_{p^{n}, \text { nr }}^{q+1}(F)$ de degré 0 , définie par

$$
w \otimes \bar{b}_{1} \otimes \ldots \otimes \bar{b}_{q} \quad \bmod J_{q}(\kappa(v)) \mapsto i(w) \cup h_{p^{n}, F}^{q}\left(b_{1}, \ldots, b_{q}\right)
$$

D'autre part, on a une inclusion $\psi: H_{p^{n}}^{q}(\kappa(v)) \rightarrow H_{p^{n}, \text { nr }}^{q+1}(F)$ de degré 1 , définie par

$$
w \otimes \bar{b}_{2} \otimes \ldots \otimes \bar{b}_{q} \quad \bmod J_{q-1}(\kappa(v)) \mapsto i(w) \cup h_{p^{n}, F}^{q}\left(\pi, b_{2}, \ldots, b_{q}\right) .
$$

Ici, $w \in W_{n}(\kappa(v)), \pi$ est une uniformisante fixe de $F, b_{i} \in \mathcal{O}_{v}^{\times}, h_{p^{n}, F}^{q}$ est le symbole galoisien (2.2) et $i$ est l'homomorphisme canonique

$$
W_{n}(\kappa(v)) /\left\{w^{(p)}-w \mid w \in W_{n}(\kappa(v))\right\} \stackrel{\varphi}{\cong} H^{1}\left(\kappa(v), \mathbb{Z} / p^{n} \mathbb{Z}\right) \hookrightarrow H_{p^{n}}^{1}(F) .
$$

La dernière injection ci-dessus est celle de la suite (2.5), et l'isomorphisme $\varphi$ vient du théorème 90 d'Hilbert additif appliqué à la suite exacte de cohomologie associée à la suite exacte de Witt [Wit, §5] :

$$
0 \longrightarrow \mathbb{Z} / p^{n} \mathbb{Z} \longrightarrow W_{n}\left(\kappa(v)_{s}\right) \stackrel{x^{(p)}-x}{\longrightarrow} W_{n}\left(\kappa(v)_{s}\right) \longrightarrow 0 .
$$

Kato démontre donc que $i^{*} \oplus \psi$ donne un isomorphisme

$$
H_{p^{n}}^{q+1}(\kappa(v)) \oplus H_{p^{n}}^{q}(\kappa(v)) \cong H_{p^{n}, \mathrm{nr}}^{q+1}(F) .
$$

- Le cas d'égale caractéristique $0(\operatorname{car}(F)=\operatorname{car}(\kappa(v))=0)$ est comme le cas modéré. En effet, $H_{p^{n}, \mathrm{nr}}^{q+1}(F)=H_{p^{n}}^{q+1}(F)$, puisque selon la suite scindée (2.3), on a

$$
H_{p^{n}}^{q+1}\left(F_{\mathrm{nr}}\right) \cong H_{p^{n}}^{q+1}\left(\kappa(v)_{s}\right) \oplus H_{p^{n}}^{q+1}\left(\kappa(v)_{s}\right)=0 .
$$

- Le cas d'égale caractéristique $p(\operatorname{car}(F)=\operatorname{car}(\kappa(v))=p)$ est décrit par Izhboldin [Izh, Prop. 6.8]. Le morphisme $i^{*}: H_{p^{n}}^{q+1}(\kappa(v)) \rightarrow H_{p^{n}, \mathrm{nr}}^{q+1}(F)$ est défini par

$$
\bar{w} \otimes \bar{b}_{1} \otimes \ldots \otimes \bar{b}_{q} \quad \bmod J_{q}(\kappa(v)) \mapsto w \otimes b_{1} \otimes \ldots \otimes b_{q} \quad \bmod J_{q}(F) .
$$

D'autre part il y a un morphisme $\psi: H_{p^{n}}^{q}(\kappa(v)) \rightarrow H_{p^{n}, \text { nr }}^{q+1}(F)$, défini par

$$
\bar{w} \otimes \bar{b}_{2} \otimes \ldots \otimes \bar{b}_{q} \quad \bmod J_{q-1}(\kappa(v)) \mapsto w \otimes \pi \otimes b_{2} \otimes \ldots \otimes b_{q} \quad \bmod J_{q}(F),
$$

où $\pi$ est de nouveau une uniformisante fixe de $F, b_{i} \in \mathcal{O}_{v}^{\times}, w=\left(a_{1}, \ldots, a_{n}\right) \in$ $W_{n}\left(\mathcal{O}_{v}\right)$ et $\bar{w}=\left(\bar{a}_{1}, \ldots, \bar{a}_{n}\right)$ son résidu dans $W_{n}(\kappa(v))$. Izhboldin démontre que $i^{*} \oplus \psi$ induit un scindage de $H_{p^{n}, \mathrm{nr}}^{q+1}(F)$. 
(d) Définition du R-module de cycles $\mathcal{H}_{p^{n}, L}^{*}$ - Maintenant, on a assez d'information afin de définir notre module de cycles envisagé.

Définition 4.3. Soient $(K, R, k)$ un p-triplet de Cohen avec une extension de Cohen finie, galoisienne $(L, S, \bar{L})$. Alors pour tout entier $n>0$, on définit $\mathcal{H}_{p^{n}, L}^{*}=\left(\mathcal{H}_{p^{n}, L}^{i}(F)\right)_{i>0}$ le module de cycles de base $R$ avec

$$
\mathcal{H}_{p^{n}, L}^{j+1}(F)=H_{p^{n}, L}^{j+1}(F):= \begin{cases}\operatorname{ker}\left(H_{p^{n}}^{j+1}(F) \rightarrow H_{p^{n}}^{j+1}\left(F \otimes_{K} L\right)\right) & \text { si } F \in K-\mathfrak{c o r p s}, \\ \operatorname{ker}\left(H_{p^{n}}^{j+1}(F) \rightarrow H_{p^{n}}^{j+1}\left(F \otimes_{k} \bar{L}\right)\right) & \text { si } F \in k \text {-corps. }\end{cases}
$$

Remarque 4.4 - $\grave{A}$ noter que pour $F \in K$-corps les groupes de cohomologie sont des groupes de cohomologie galoisienne usuelles.

Remarque 4.5 - Si $\left(L_{1}, S_{1}, \bar{L}_{1}\right)$ et $\left(L_{2}, S_{2}, \bar{L}_{2}\right)$ sont deux extensions de Cohen finies galosiennes de $(K, R, k)$, il existe une extension de Cohen finie galoisienne $(L, S, \bar{L})$ de $(K, R, k)$ tel qu'elle est une extension de Cohen commune de $\left(L_{1}, S_{1}, \bar{L}_{1}\right)$ et $\left(L_{2}, S_{2}, \bar{L}_{2}\right)$. Alors, ils existent des injections $\mathcal{H}_{n, L_{1}}^{*} \rightarrow \mathcal{H}_{n, L}^{*}$ et $\mathcal{H}_{n, L_{2}}^{*} \rightarrow \mathcal{H}_{p^{n}, L}^{*}$. Le choix de $L$ n'a donc pas grande importance.

Il faut démontrer que cette définition définit bien un module de cycles. Pour cela, on a tout d'abord besoin des quatre données D1-D4 (voir \$2.2 (a)). Les données D1, D2 et D3 se déroulent seulement en égales caractéristiques. Pour la donnée D4, on peut rencontrer des caractéristiques mixtes.

Par cette remarque, la fonctorialité (D1) suit immédiatement. Soit $E$ une extension finie de $F$ de trace $\operatorname{Tr}_{E / F}$. Alors, $E \otimes_{F} C_{p^{n}}^{q}(F) \cong C_{p^{n}}^{q}(E)$, et on définit une trace de $C_{p^{n}}^{q}(E)$ par la composition

$$
C_{p^{n}}^{q}(E) \cong E \otimes_{F} C_{p^{n}}^{q}(F) \stackrel{\operatorname{Tr}_{E / F} \otimes \mathrm{id}}{\longrightarrow} F \otimes_{F} C_{p^{n}}^{q}(F) \cong C_{p^{n}}^{q}(F) .
$$

Celle-ci s'étend alors à une défintion pour la réciprocité (D2) aux groupes de cohomologie $H_{p^{n}, L}^{q+1}(F)$.

(e) Structure de $K_{m}(F)$-module (D3) - Si car $(F)=0$ (i.e. $F$ est une extension de $K$ ), la structure de $K_{m}(F)$-module est définie comme dans le cas modéré. $\mathrm{Si} \operatorname{car}(F)=p$ (i.e. $F$ est une extension de $k$ ), cette structure est inspirée par le symbole différentiel au lieu du symbole galoisien. Pour tout $m \geq 1$,

$$
\rho_{F}^{m}: K_{m}(F) \rightarrow \Omega_{F}^{m}, \quad \text { défini par } \quad\left\{x_{1}, \ldots, x_{m}\right\} \mapsto \frac{d x_{1}}{x_{1}} \wedge \ldots \wedge \frac{d x_{m}}{x_{m}},
$$

est un homomorphisme. En effet, $d(a b)=b d(a)+a d(b)$ implique $\frac{d(a b)}{a b}=\frac{d a}{a}+\frac{d b}{b}$, et si $a+b=1$, on a $\frac{d a}{a} \wedge \frac{d b}{b}=0$, puisque $d a+d b=0\left(a, b \in k^{\times}\right)$. Alors, $\rho_{F}^{m}$ induit une application $K_{m}(F) / p K_{m}(F) \rightarrow \Omega_{F}^{m}$ parce que $\operatorname{car}(F)=p$ (et donc $d x^{p}=0$ ). De plus, l'image est contenue dans $\nu_{1}(m)_{F}$. Le symbole différentiel est $h_{1, F}^{m}: K_{m}(F) / p K_{m}(F) \rightarrow \nu_{1}(m)_{F}$. Le Théorème de Bloch-Kato-Gabber démontre que c'est un isomorphisme [BK, Thm. 2.1].

Inspiré par la définition du symbole différentiel, on peut proposer la structure de $K_{m}(F)$-module comme suit :

$$
\begin{aligned}
\rho_{p^{n}, F}^{m}: K_{m}(F) \times H_{p^{n}}^{q+1}(K) & \rightarrow H_{p^{n}}^{q+m+1}(F): \\
\left(\left\{x_{1}, \ldots, x_{m}\right\}, w \otimes b_{1} \otimes \ldots \otimes b_{q}\right) & \mapsto w \otimes x_{1} \otimes \ldots \otimes x_{m} \otimes b_{1} \otimes \ldots \otimes b_{q} .
\end{aligned}
$$


Les mêmes arguments que ci-dessus garantissent qu'elle est bien définie. Pour $a \in K_{m}(F)$ et $b \in H_{p^{n}}^{q+1}(F)$, on note la multiplication scalaire par $a \cdot b:=\rho_{p^{n}, F}^{m}(a, b)$. Cette structure se restreint à une structure de $K_{m}(F)$-module sur $\left(H_{p^{n}, L}^{q+1}(F)\right)_{q \geq 0}$ pour $(L, S, \bar{L})$ comme dans la Définition 4.3. En effet, si $b \in J_{q}(F \otimes \bar{L})$, on a $a \cdot b \in J_{q+m}(F \otimes \bar{L})$ pour tout $a \in K_{m}(F)$.

(f) Le résidu et une suite exacte - Puis, il faut l'existence d'un résidu (la donnée D4), et on veut aussi généraliser la suite exacte (2.5).

Proposition 4.6. Soient $(K, R, k)$ un p-triplet de Cohen et $(L, S, \bar{L})$ une extension de Cohen finie galoisienne. Pour tout $R$-triplet de valuation $\left(F, \mathcal{O}_{v}, \kappa(v)\right)$ et pour tous entiers $n>0$ et $q \geq 0$, on a une suite exacte scindée

$$
0 \rightarrow H_{p^{n}, L}^{q+1}(\kappa(v)) \rightarrow H_{p^{n}, L}^{q+1}(F) \rightarrow H_{p^{n}, L}^{q}(\kappa(v)) \rightarrow 0 .
$$

Démonstration. On a certainement deux versions de la suite (4.4) :

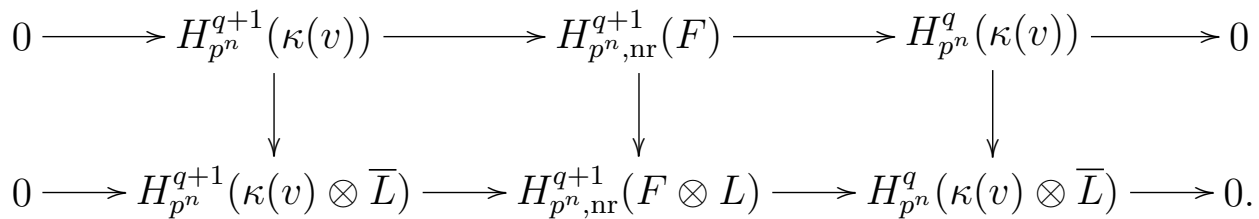

Puisque les suites sont scindées et les scindages respectent ce diagramme commutatif, la suite exacte scindée suit du lemme du serpent.

Remarque 4.7 - Les résidus pour un $R$-corps $F$ complet de valuation discrète $v$ sont donc définis par cette suite. Si $F$ n'est pas complet, on prend $\hat{F}$ un completé par rapport à $v$. Le corps résiduel de $\hat{F}$ est égal au corps résiduel $\kappa(v)$ de $F$, et alors le résidu est défini par la composition

$$
H_{p^{n}, L}^{i+1}(F) \rightarrow H_{p^{n}, L}^{i+1}(\hat{F}) \rightarrow H_{p^{n}, L}^{i}(\kappa(v)) .
$$

On a donc introduit les quatre données des modules de cycles, et en plus on a une suite qui nous permet de faire la même construction que dans le cas modéré. Pour la vérification détaillée des règles de module de cycles, nous renvoyons à l'Appendice A.

(g) Version relative - Comme dans $\$ 2.1(\mathrm{~d})$, nous définissons de nouveau des modules de cycles relatifs à base de l'isomorphisme (4.3) et de l'action de la $K$-théorie - comparable avec la présentation (2.6) du module de cycle relatif moderé.

Définition 4.8. Soient $(K, R, k)$ un p-triplet de Cohen, $A$ une $K$-algèbre simple centrale de $\operatorname{ind}_{K}(A)=p^{n}$ et $\bar{A}$ la $k$-algèbre simple centrale résiduelle. Soit $(L, S, \bar{L})$ une extension de Cohen finie galoisienne de $(K, R, k)$ tel que $\bar{L}$ est un corps de décomposition de $\bar{A}$. Soient $F \in R$-corps et $\left[A_{F}\right]$ la classe de $A_{F}:=A \otimes_{R} F$ dans ${ }_{p^{n}} \operatorname{Br}(F)$, alors on définit le module de cycles $\mathcal{H}_{p^{n}, L, \mathcal{A}}^{*}=\left(H_{p^{n}, L, A}^{j}\right)_{j \geq 2}$ de base $R$ par

$$
H_{p^{n}, L, A}^{j+1}(F):=H_{p^{n}, L}^{j+1}(F) /\left(K_{j-1}(F) \cdot\left[A_{F}\right]\right) .
$$


Remarque 4.9 - Le choix de $\bar{L}$ est bien possible dû au théorème de Wedderburn qui donne une extension finie, séparable $\bar{L}^{\prime}$ qui déploie $\bar{A}$. On obtient $\bar{L}$ en prenant une extension finie de $\bar{L}^{\prime}$ tel que l'extension $\bar{L} / k$ est galoisienne. Puis, on associe donc un triplet de Cohen $(L, S, \bar{L})$ à $\bar{L}$.

On peut même supposer que $\bar{L}$ est une extension cyclique de $k$. En effet, le théorème d'Albert [Alb, Thm. 18] dit que toute $k$-algèbre simple centrale de degré $p^{n}$ est Braueréquivalente à une $k$-algèbre cyclique, et la définition de $\mathbf{S K}_{1}(A)$ ne dépend pas du représentant de la classe de Brauer de $A$ (isomorphisme de Morita [GS, Lem. 2.8.6]).

Le fait qu'on choisit $\bar{L}$ comme corps de décomposition de $\bar{A}$ est afin de garantir que la multiplication scalaire se trouve dans $\mathcal{H}_{p^{n}, L}^{*}$. En effet, pour une extension $F$ de $k$ l'extension de base de $\bar{A}$ à $\bar{L}$ rend $\left[\bar{A}_{F}\right]$ triviale dans le groupe de Brauer, et donc également le sous-groupe $K_{j-1}(F)$. [A $\left.A_{F}\right]$. De plus, $L$ deploie $A$ parce que $\operatorname{Br}(\bar{L}) \rightarrow \operatorname{Br}(L)$ est injective $(\$ 3.2(\mathrm{a})$.

Il faut donc vérifier que cette définition relative définit bien un module de cycles. On se fonde sur le fait qu'on dispose d'un module de cycles dans le cas absolu, et on vérifie que les données sont bien définies modulo les sous-groupes en jeu. Les données D1, D2 et D3 suivent plus au moins de la définition, et parce que les corps en jeu ont toujours la même caractéristique. La donnée D4 pour le cas d'égale caractéristique (du corps de valuation discrète et de son corps résiduel) suit aussi de la définition des modules de cycles et des résidus de $\mathcal{H}_{p^{n}, L}^{*}$. Il suffit donc de vérifier la donnée D4 pour le cas de caractéristique mixte. De plus, on veut géneraliser la suite exacte (4.5).

Proposition 4.10. Avec les mêmes données de la Définition 4.8, on a pour tout triplet de valuation $\left(F, \mathcal{O}_{v}, \kappa(v)\right)$ sur $R$ une suite exacte

$$
0 \rightarrow H_{p^{n}, L, A}^{q+1}(\kappa(v)) \rightarrow H_{p^{n}, L, A}^{q+1}(F) \rightarrow H_{p^{n}, L, A}^{q}(\kappa(v)) \rightarrow 0 .
$$

Démonstration. Suite à la discussion précedente, il suffit de démontrer l'énoncé dans le cas de caractéristique mixte $(\operatorname{car}(F)=0$ et $\operatorname{car}(\kappa(v))=p)$. Le but est donc de vérifier que la suite exacte (4.5) commute avec les inclusions dans un diagramme commutatif (au signe près et pour $q \geq 2$ )

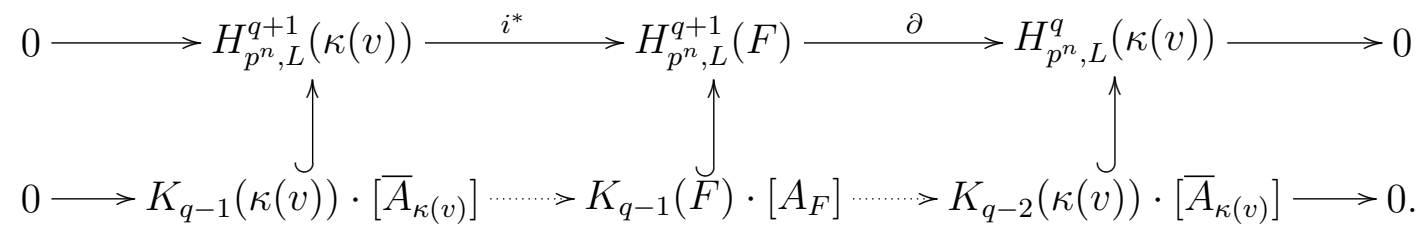

Vérifions tout d'abord qu'au signe près le diagramme

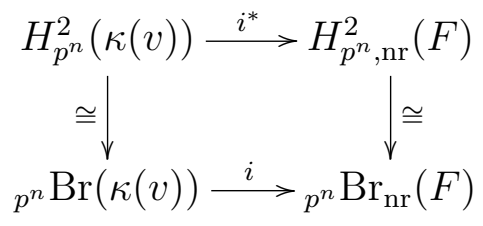

commute, où $\operatorname{Br}_{\mathrm{nr}}(F)=\operatorname{ker}\left(\operatorname{Br}(F) \rightarrow \operatorname{Br}\left(F_{\mathrm{nr}}\right)\right)$, $i^{*}$ le morphisme de la suite 4.4 et $i$ l'injection de $\$ 3.2$ (a). Alors, la vérification est un calcul immédiat, faisons-le. Soit $\bar{a} \otimes \bar{x} \in H_{p^{n}}^{2}(\kappa(v))$ avec $a \in W_{n}\left(\mathcal{O}_{v}\right)$ et $x \in \mathcal{O}_{v}^{\times}$. Alors,

$$
i^{*}(\bar{a} \otimes \bar{x})=\left((\tau(y) / y)^{\sigma(b)-b}\right)_{\sigma, \tau} \in H_{p^{n}}^{2}(F)
$$


avec $y^{p}=x$ et $a=b^{p}-b$ pour $y \in W_{n}\left(F_{\mathrm{nr}}\right)$ et $b \in F_{\mathrm{nr}}^{\times}$. Alors, l'image dans ${ }_{p^{n}} H^{2}\left(F, F_{s}^{\times}\right) \cong$ $p^{n} \operatorname{Br}(F)$ est représentée par la même expression. Par ailleurs, l'image de $\bar{a} \otimes \bar{x} \in H_{p^{n}}^{2}(\kappa(v))$ dans $p^{n} H^{2}\left(\kappa(v), \kappa(v)_{s}^{\times}\right) \cong p^{n} \operatorname{Br}(\kappa(v))$ est $c:=\left((\sigma(\bar{y}) / \bar{y})^{\tau(\bar{b})-\bar{b}}\right)_{\sigma, \tau}$. Alors,

$$
i(c)=\left((\sigma(y) / y)^{\tau(b)-b}\right)_{\sigma, \tau} \in H_{p^{n}}^{2}(F) .
$$

Parce que $i^{*}$ est défini par un cup-produit, il est égal à $i^{*}(\bar{a} \otimes \bar{x})$ à signe près.

La restriction de (4.6) aux sous-groupes donne le diagramme commutatif (au signe près)

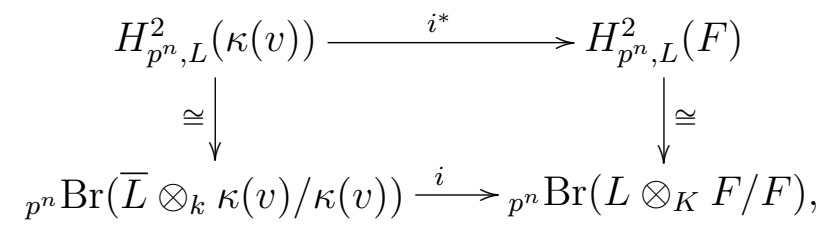

avec

$$
\begin{aligned}
\operatorname{Br}\left(L \otimes_{K} F / F\right) & =\operatorname{ker}\left[\operatorname{Br}(F) \rightarrow \operatorname{Br}\left(L \otimes_{K} F\right)\right] \quad \text { et } \\
\operatorname{Br}\left(\bar{L} \otimes_{k} \kappa(v) / \kappa(v)\right) & =\operatorname{ker}\left[\operatorname{Br}(\kappa(v)) \rightarrow \operatorname{Br}\left(\bar{L} \otimes_{k} \kappa(v)\right)\right] .
\end{aligned}
$$

La démonstration de ce théorème suit tout directement de ce fait parce que les morphismes $i^{*}, \partial$ et la retraction $\psi$ (voir (c) respectent la structure de modules de $K$-théorie, et parce que le signe disparaît au niveau des groupes de quotient. Donc,

$$
\begin{aligned}
i^{*}\left(K_{q-1}(\kappa(v)) \cdot\left[\bar{A}_{\kappa(v)}\right]\right) & =i_{K}^{*}\left(K_{q-1}(\kappa(v))\right) \cdot i^{*}\left(\left[\bar{A}_{\kappa(v)}\right]\right) \subset K_{q-1}(F) \cdot\left[A_{F}\right], \\
\partial\left(K_{q-1}(F) \cdot\left[A_{F}\right]\right) & =\partial_{K}\left(K_{q-1}(F)\right) \cdot\left[\bar{A}_{\kappa(v)}\right]=K_{q-2}(\kappa(v)) \cdot\left[\bar{A}_{\kappa(v)}\right] \text { et } \\
\psi\left(K_{q-2}(\kappa(v)) \cdot\left[\bar{A}_{\kappa(v)}\right]\right) & =\psi_{K}\left(K_{q-2}(\kappa(v))\right) \cdot i^{*}\left(\left[\bar{A}_{\kappa(v)}\right]\right) \subset K_{q-1}(F) \cdot\left[A_{F}\right] .
\end{aligned}
$$

Ici,

$$
\begin{array}{lll}
i_{K}^{*}: K_{q-1}(\kappa(v)) \rightarrow K_{q-1}(F), \quad \text { défini par } & \left\{\bar{x}_{1}, \ldots, \bar{x}_{q-1}\right\} \mapsto\left\{x_{1}, \ldots, x_{q-1}\right\}, \\
\partial_{K}: K_{q-1}(F) \rightarrow K_{q-2}(\kappa(v)), \quad \text { défini par } \quad\left\{\begin{array}{l}
\left\{x_{1}, \ldots, x_{q-1}\right\} \mapsto 0, \\
\left\{\pi, x_{2}, \ldots, x_{q-2}\right\} \mapsto\left\{\bar{x}_{2}, \ldots, \bar{x}_{q-2}\right\},
\end{array}\right. \\
\psi_{K}: K_{q-2}(\kappa(v)) \rightarrow K_{q-1}(F), \quad \text { défini par } \quad\left\{\bar{x}_{2}, \ldots, \bar{x}_{q-2}\right\} \mapsto\left\{\pi, x_{2}, \ldots, x_{q-2}\right\},
\end{array}
$$

pour $x_{i} \in \mathcal{O}_{v}^{\times}$et $\pi$ une uniformisante de $F$ pour la valuation $v$.

Notons que cette suite exacte satisfait une propriété analogue au Corollaire 3.8, puisque dans ce cas les scindages sont aussi donnés par un choix d'uniformisante qui est canonique pour des anneaux de Cohen - voir les définitions dans la Remarque 4.2.

Corollaire 4.11. Prenons les mêmes données de la Définition 4.8 et $\left(F, \mathcal{O}_{v}, \kappa(v)\right)$ une extension de Cohen de $(K, R, k)$. Notons $u: k \rightarrow \kappa(v)$ l'inclusion. Le Théorème 3.6 (i) fournit un homomorphisme local $v: R \rightarrow \mathcal{O}_{v}$, alors $v$ définit pour tous entiers $i, n \geq 0$ un homomorphisme de suites exactes scindées:

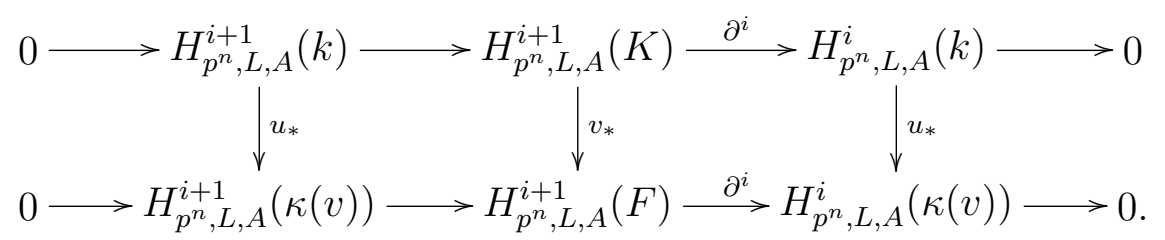

De plus, $v_{*}$ ne dépend pas du choix de $v$. Si $k=k^{\prime}$, on a un isomorphisme canonique $H_{n, B_{K}}^{i+1}(K) \cong H_{n, B_{K}}^{i+1}(F)$. 


\subsection{Le relèvement}

Avant de relever, on démontre un résultat analogue à celui de Merkurjev (Proposition 3.13). Ce sera un corollaire immédiat du Théorème de Kahn suivant qui utilise des groupes de cohomologie de Zariski avec leur variante réduite :

$$
\tilde{H}_{Z a r}^{0}\left(\mathbf{G}, H_{p^{n}}^{3}\right) \cong H_{Z a r}^{0}\left(\mathbf{G}, H_{p^{n}}^{3}\right) / H_{p^{n}}^{3}(k) .
$$

Théorème 4.12 (Kahn [Kah]). Soient $k$ un corps de car $(k)=p, \boldsymbol{G}$ un k-groupe algébrique simplement connexe, absolument $k$-simple et $\overline{\boldsymbol{G}}:=\boldsymbol{G} \times{ }_{k} k_{s}$ et $n>0$ entier. Si $C^{2}(G)=0$, on a une injection

$$
\tilde{H}_{Z a r}^{0}\left(\boldsymbol{G}, H_{p^{n}}^{3}\right) \hookrightarrow H_{Z a r}^{0}\left(\overline{\boldsymbol{G}}, H_{p^{n}}^{3}\right)
$$

Remarque 4.13 - Dans l'enoncé (et dans la suite), $G$ (resp. $\bar{G}$ ) signifie $\mathbf{G}(k)$ (resp. $\overline{\mathbf{G}}\left(k_{s}\right)$ ).

Démonstration. Soit $\Gamma=\Gamma_{k}$ le groupe de Galois absolu de $k$. Utilisant la cohomologie motivique à la Lichtenbaum, Kahn construit un morphisme (ibid., première suite exacte p. 406)

$$
\tilde{H}_{\mathrm{Zar}}^{0}\left(\mathbf{G}, H_{p^{n}}^{3}\right) \rightarrow \mathbb{H}^{5}\left(\overline{\mathbf{G}} / k_{s}, \Gamma(2)\right)^{\Gamma}
$$

dont le noyau est contenu dans $H^{1}\left(F, H_{\text {Zar }}^{1}\left(\overline{\mathbf{G}}, \mathcal{K}_{2}\right)\right)$. Ici, $\mathbb{H}^{5}\left(\mathbf{G} / k_{s}, \Gamma(2)\right)$ est un groupe d'hypercohomologie défini par Kahn comme le (5-ième) groupe de hypercohomologie étale d'un complexe relatif basé sur le complexe $\Gamma(2)$ de Lichtenbaum Lic], et $\mathcal{K}_{2}$ est le faisceau de Zariski associé au préfaisceau $U \mapsto K_{2}^{Q}(U)$ (avec $K_{2}^{Q}$ la $K$-théorie de Quillen). Afin de définir le morphisme, il faut que $H_{\mathrm{Zar}}^{0}\left(\overline{\mathbf{G}}, \mathcal{K}_{2}\right) \cong K_{2}^{Q}\left(k_{s}\right)$ : fait qui est dû à EsnaultKahn-Levine-Viehweg [EKLV, Prop. 3.20]. Puisque $H_{\text {Zar }}^{1}\left(\overline{\mathbf{G}}, \mathcal{K}_{2}\right)=\mathbb{Z}$ [Gil1, Prop. 1'], le morphisme (4.8) est injectif. (Voir les références pour plus de détails sur ces objets qu'on utilise ici jusque commes objets auxiliaires.) Puisque $\mathrm{CH}^{2}(\bar{G})^{\Gamma}=0$ [EKLV, Prop. 3.20], l'enoncé suit alors de l'injection suivante de Kahn (ibid., suite exacte (18) p. 404) :

$$
\mathbb{H}^{5}\left(\overline{\mathbf{G}} / k_{s}, \Gamma(2)\right)^{\Gamma} \hookrightarrow H_{\mathrm{Zar}}^{0}\left(\overline{\mathbf{G}}, H_{p^{n}}^{3}\right) .
$$

Corollaire 4.14. Soient $k$ un corps de caractéristique $p>0, L$ une extension finie séparable de $k$ et $\boldsymbol{G}$ un $k$-groupe algébrique lisse semi-simple simplement connexe absolument $k$-simple tel que $C H^{2}(G)=0$. Alors, $\operatorname{Inv}^{3}\left(\boldsymbol{G}, \mathcal{H}_{p^{n}, L}^{3}\right)=0$ pour tout entier $n>0$.

Remarque 4.15 - Ici, $\mathcal{H}_{p^{n}, L}^{3}$ est le module de cycles de la Définition 4.3 restreint à $k$-corps suivant $\$ 2.1$ (b). On utilise ici $L$ au lieu de $\bar{L}$ qui apparaît dans la Définition 4.3 pour ne pas allourdir la notation.

Démonstration. Il suffit de démontrer que $\tilde{A}^{0}\left(\mathbf{G}, \mathcal{H}_{p^{n}, L}^{3}\right)=0$. Puisque Rost démontre que $A^{i}\left(\mathbf{G}, M_{j}\right) \cong H_{\mathrm{Zar}}^{i}\left(\mathbf{G}, M_{j}\right)$ pour un module de cycles $M$ et des entiers $i, j$ [Ros1, Cor. 6.16], il suffit de démontrer que $\tilde{H}_{\text {Zar }}^{0}\left(\mathbf{G}, \mathcal{H}_{p^{n}, L}^{3}\right)=0$.

Soit donc $x \in \tilde{H}_{\mathrm{Zar}}^{0}\left(\mathbf{G}, \mathcal{H}_{p^{n}, L}^{3}\right) \subset \tilde{H}_{\mathrm{Zar}}^{0}\left(\mathbf{G}, \mathcal{H}_{p^{n}}^{3}\right)$. On sait que $H_{p^{n}}^{3}(k(\mathbf{G})) \rightarrow H_{p^{n}}^{3}\left(k_{s}(\mathbf{G})\right)$ se factorise à travers $H_{p^{n}}^{3}(k(\mathbf{G}) \otimes L)$. Alors, $x \in \operatorname{ker}\left[H_{p^{n}}^{3}(k(\mathbf{G})) \rightarrow H_{p^{n}}^{3}\left(k_{s}(\mathbf{G})\right)\right]$, puisque $x \in H_{p^{n}, L}^{3}(k(\mathbf{G}))$, et donc $x \in \operatorname{ker}\left[\tilde{H}_{\mathrm{Zar}}^{0}\left(\mathbf{G}, \mathcal{H}_{p^{n}}^{3}\right) \rightarrow \tilde{H}_{\mathrm{Zar}}^{0}\left(\overline{\mathbf{G}}, \mathcal{H}_{p^{n}}^{3}\right)\right]$. Le Théorème 4.12 donne que $x=0$. 
Les arguments utilisés dans la démonstration du Théorème 3.11 sont de type homologique et sont bien transférables au cas sauvage remplaçant la Proposition 3.13 par le Corollaire 4.14, On obtient donc le théorème suivant.

Théorème 4.16. Soient $k$ un corps de $\operatorname{car}(k)=p>0, A$ une $k$-algèbre simple centrale de $\operatorname{ind}_{k}(A)=p^{n}$ et $\bar{L}$ une extension finie, galoisienne de $k$ qui déploie $A$. Soit $(K, R, k)$ un triplet de Cohen associé à $k$ et $(L, S, \bar{L})$ un triplet de Cohen associé à $\bar{L}$. Soient $B$ la $R$-algèbre d'Azumaya relevée et $\rho^{\prime} \in \operatorname{Inv}^{4}\left(\boldsymbol{S K}_{1}\left(B_{K}\right), \mathcal{H}_{p^{n}, L, \mathcal{B}_{K}}^{*}\right)$. Alors, il existe un unique $\rho \in \operatorname{Inv}^{4}\left(\boldsymbol{S K}_{1}(A), \mathcal{H}_{p^{n}, L, \mathcal{A}}^{*}\right)$, que l'on appelle l'invariant spécialisé de $\rho^{\prime}$, tel que pour toute extension de Cohen $\left(K^{\prime}, R^{\prime}, k^{\prime}\right)$ de $(K, R, k)$ le diagramme suivant commute :

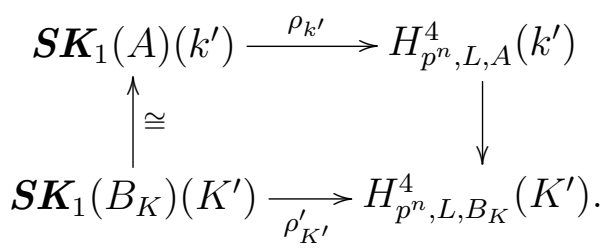

Remarque 4.17 - De nouveau, les modules de cycles $\mathcal{H}_{p^{n}, L, \mathcal{B}_{K}}^{*}:=\left(\mathcal{H}_{p^{n}, L, \mathcal{B}_{K}}^{j}\right)_{j \geq 2}$ de base $K$ et $\mathcal{H}_{p^{n}, L, \mathcal{A}}^{*}:=\left(\mathcal{H}_{p^{n}, L, \mathcal{A}}^{j}\right)_{j \geq 2}$ de base $k$ sont les modules de cycles évidents. Il sont les modules de cycles restreints de la module de cycles $\mathcal{H}_{p^{n}, L, \mathcal{B}_{K}}^{*}$ de base $R$ respectivement à $K$ et à $k$ (selon $2.2(\mathrm{~b})$.

Démonstration. Afin de généraliser la démonstration du Théorème 3.11, il faut généraliser le Corollaire 3.14. Il suffit donc de définir un diagramme comme 3.2 parce que les autres arguments sont une simple chasse au diagramme transférable au cas sauvage. Soit donc $\mathbf{G}=\mathbf{S L}_{1}(A)$. On considère le diagramme suivant dont les colonnes sont exactes, et dont la ligne centrale est exacte (Corollaire 4.14) :

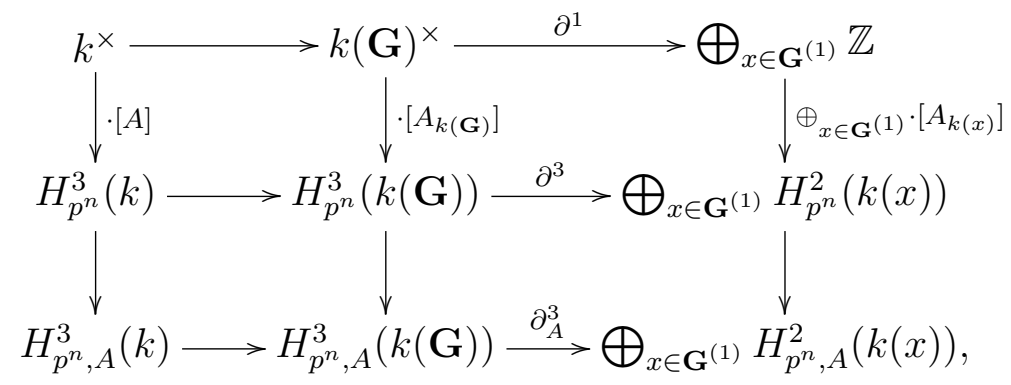

De nouveau, l'application $\partial^{1}$ est le morphisme diviseur, et parce que $\operatorname{Pic}(\mathbf{G})=0$ [San, Lem. 6.9], $\partial^{1}$ est surjective. Il faut aussi noter que $\mathrm{CH}^{2}(G)=0$ puisque $\mathbf{G}$ est désormais une forme intérieure de $\mathbf{S L}_{m}(k)$ avec $m=\operatorname{deg}_{k}(A)[\mathrm{Pan}$. La même chasse au diagramme et la même construction que dans le cas modéré finissent la démonstration.

On peut donc en déduire que l'invariant de Suslin se généralise.

Corollaire 4.18. Sous les mêmes conditions que dans le Théorème 4.16 l'invariant de Suslin $\rho_{\text {Sus }, B_{K}}$ induit un unique invariant dans $\operatorname{Inv}^{4}\left(\boldsymbol{S K}_{1}(A), \mathcal{H}_{p^{n}, L, \mathcal{A}}^{*}\right)$ (satisfaisant la propriété de relèvement), que l'on appelle l'invariant de Suslin $\rho_{\text {Sus }, A} d e A$. 
Démonstration. Il faut démontrer que $\rho=\rho_{\text {Sus, } B_{K}}$ a ses valeurs dans $\mathcal{H}_{p^{n}, L, B_{K}}^{4}$. Ceci suit du diagramme commutatif

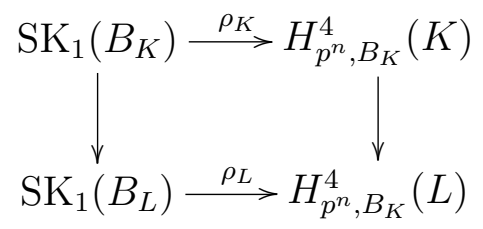

et de la trivialité de $\mathrm{SK}_{1}\left(B_{L}\right)$ ( $L$ déploie $\left.B_{K}\right)$.

\subsection{Le cas général}

Soient $k$ un corps de caractéristique $p>0$ et $A$ une $k$-algèbre simple centrale d'indice arbitraire. On sait qu'il existe une $k$-algèbre à division $D$, unique à isomorphisme près, qui est Brauer-équivalente à $A$. Le théorème de décomposition de Brauer [GS, Prop. 4.5.16]

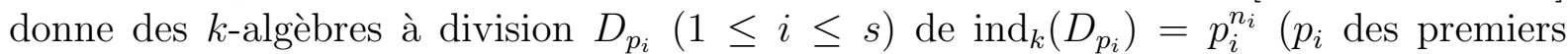
différents) tel que $D \cong D_{p_{1}} \otimes \ldots \otimes D_{p_{s}}$. Cet isomorphisme implique un isomorphisme (ibid, Ch. 4, Ex. 9)

$$
\mathbf{S K}_{1}(A) \cong \mathbf{S K}_{1}(D) \cong \mathbf{S K}_{1}\left(D_{p_{1}}\right) \oplus \ldots \oplus \mathbf{S K}_{1}\left(D_{p_{s}}\right) .
$$

Mettons $D_{\not p p}=\bigotimes_{p_{i} \neq p} D_{p_{i}}$. On a donc a fortiori un isomorphisme $\mathbf{S K}_{1}(A) \cong \mathbf{S K}_{1}\left(D_{p}\right) \oplus$ $\operatorname{SK}_{1}\left(D_{\not p}\right)$. Posons aussi $A_{p}:=D_{p}$ et $A_{\not p}:=D_{\not p}$.

Afin de définir l'invariant de Suslin en toute géneralité, nous allons coller l'invariant modéré (Théorème 3.11) et sauvage (Théorème 4.16) avec cet isomorphisme de $\mathbf{S K}_{1}(A)$. Il faut donc recoller aussi les deux modules de cycles afin d'obtenir le suivant.

Définition 4.19. Soient $(K, R, k)$ un p-triplet de Cohen, $A$ une $K$-algèbre simple centrale de $\operatorname{ind}_{K}(A)=r=p^{n} m(p \nmid m)$ et $\bar{A}$ la $k$-algèbre simple centrale résiduelle. Soit $\bar{L}$ une extension finie galoisienne de $k$ tel qu'elle est un corps de décomposition de $\bar{A}_{p}$ et $(L, S, \bar{L})$ un $p$-triplet de Cohen associé. On définit le module de cycles suivant de base $R$ :

$$
\mathcal{H}_{r, L, \mathcal{A}}^{*}:=\mathcal{H}_{m, \mathcal{A}_{\not p}}^{*} \oplus \mathcal{H}_{p^{n}, L, \mathcal{A}_{p}}^{*}
$$

Utilisant les Théorèmes 3.11 et 4.16 , on obtient le théorème suivant.

Théorème 4.20. Soient $k$ un corps de $\operatorname{car}(k)=p>0, A$ une $k$-algèbre simple centrale de $\operatorname{ind}_{k}(A)=r$ et $\bar{L}$ une extension finie de $k$ qui déploie $A_{p}$. Soient $(K, R, k)$ un triplet de Cohen associé à $k$ et $(L, S, \bar{L})$ un triplet de Cohen associée à $\bar{L}$. Soient $B$ la $R$ algèbre d'Azumaya relevée et $\rho^{\prime} \in \operatorname{Inv}^{4}\left(\boldsymbol{S K}_{1}\left(B_{K}\right), \mathcal{H}_{r, L, \mathcal{B}_{K}}^{*}\right)$. Alors, il existe un unique $\rho \in \operatorname{Inv}^{4}\left(\boldsymbol{S K}_{1}(A), \mathcal{H}_{r, L, \mathcal{A}}^{*}\right)$, que l'on appelle l'invariant spécialisé de $\rho^{\prime}$, tel que pour toute extension de Cohen $\left(K^{\prime}, R^{\prime}, k^{\prime}\right)$ de $(K, R, k)$ le diagramme suivant commute:

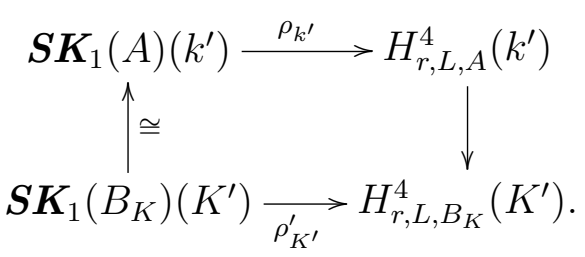

En général on sait donc définir un invariant de Suslin de $\mathbf{S K}_{1}(A)$.

Corollaire 4.21. Sous les mêmes conditions que dans le Théorème 4.20, l'invariant de Suslin $\rho_{\text {Sus }, B_{K}}$ induit un unique invariant dans $\operatorname{Inv}^{4}\left(\boldsymbol{S K}_{1}(A), \mathcal{H}_{r, L, A}^{*}\right)$ (satisfaisant la propriété de relèvement), ce que l'on appelle l'invariant de Suslin $\rho_{\text {Sus, } A} d e A$. 


\section{Remarques finales}

Finissons ce texte par donner quelques remarques. Notons tout d'abord que KnusMerkurjev-Rost-Tignol ont également défini un invariant de $\mathbf{S K}_{1}(A)$ pour une algèbre de biquaternions $A$ en caractéristique quelconque [KMRT, SS17]. L'auteur rapporte que les deux invariants sont liés. Il étudiera ce sujet dans un prochain papier [Wou] utilisant le fait qu'ils sont les mêmes dans le cas modéré [KMRT, Notes §17], [Sus2, Thm. 4]. Commentons maintenant sur un autre point de vue sur la construction et puis sur quelques réflexions de la conjecture de Suslin.

\subsection{Autre point de vue}

Il y a une autre façon de regarder à la construction utilisant les groupes $A^{i}, \tilde{A}^{0}$ et $A_{\text {mult }}^{0}$ de $\$ 2.2(\mathrm{c})$ et $\$ 2.3(\mathrm{~b})$. Soient $(K, R, k)$ un $p$-triplet de Cohen, $A$ une $k$-algèbre simple centrale de $\operatorname{ind}_{k}(A)=p^{n}, B$ la $R$-algèbre d'Azumaya relevée, $(L, S, \bar{L})$ une extension de Cohen finie galoisienne de $(K, R, k)$ tel que $\bar{L}$ deploie $A$ et $\mathcal{H}^{*}:=\mathcal{H}_{n, L, B_{K}}^{*}$ le module de cycles avec base $R$ de la Définition 4.8 . Notons $\mathcal{G}:=\mathbf{S L}_{1}(B)$ qui est défini, de façon analogue que $\mathbf{S L}_{1}\left(B_{K}\right)$, avec une norme réduite sur $B$ induit par un scindage $B \otimes_{R} S \cong$ $M_{m}(S)$ - voir [Knu , Ch. III, SS1] pour plus de détails. La fibre générique $\mathcal{G}_{K}=\mathbf{S L}_{1}\left(B_{K}\right)$ est un ouvert de $\mathcal{G}$. Le fermé complémentaire $Z$ est l'image de la fibre spéciale $\mathbf{G}=\mathbf{S L}_{1}(A)$ dans $\mathcal{G}$ sous l'immersion des schémas $\psi: \mathbf{G} \rightarrow \mathcal{G}$. Pour tout entier $i \geq 0$, les points de $Z$ de codimension $i+1$ correspondent sous $\psi$ aux points de codimension $i$ dans $\mathbf{G}$. De même façon, $\operatorname{Spec}(K)$ est un ouvert de $\operatorname{Spec}(R)$ avec fermé complémentaire l'image de $\operatorname{Spec}(k)$. La suite de localisation de Rost [Ros1, §5] donne donc des suites exactes :

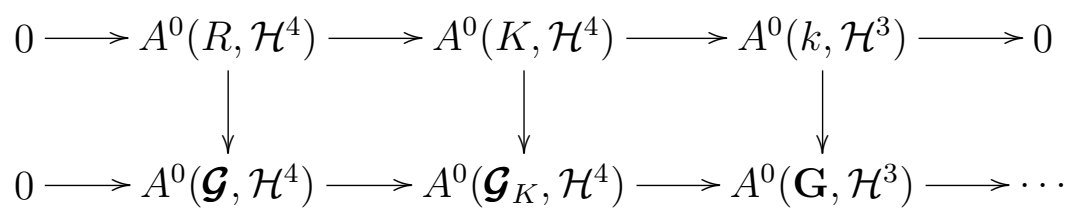

Le Corollaire 3.14 et le Corollaire 4.14 (étant généralisé à $\mathcal{H}^{*}$ dans la démonstration du

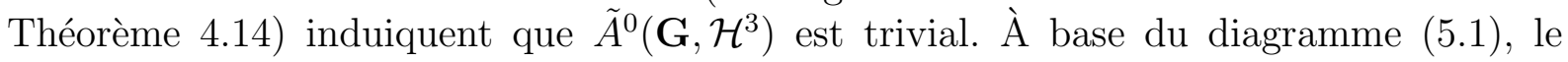
lemme du serpent donne donc un isomorphisme

$$
\tilde{A}^{0}\left(\mathcal{G}_{K}, \mathcal{H}^{4}\right) \cong \tilde{A}^{0}\left(\mathcal{G}, \mathcal{H}^{4}\right)
$$

qui respecte les éléments multiplicatifs. C'est-à-dire dû à l'isomorphisme de Merkurjev (2.7), on a également un isomorphisme

$$
\operatorname{Inv}^{4}\left(\mathcal{G}_{K}, \mathcal{H}^{*}\right) \cong \tilde{A}^{0}\left(\mathcal{G}, \mathcal{H}^{4}\right)_{\text {mult }}
$$

Le groupe à droite est défini de façon pareille que pour des groupes algébriques dans $2.3(\mathrm{~b})$. Puisque $\mathcal{H}^{*}$ a base $R$, le morphisme $\mathbf{G} \rightarrow \mathcal{G}$ de schémas donne également un morphisme

$$
A^{0}\left(\mathcal{G}, \mathcal{H}^{4}\right) \rightarrow A^{0}\left(\mathbf{G}, \mathcal{H}^{4}\right)
$$

qui donne de la même façon un morphisme d'invariants

$$
\tilde{A}^{0}\left(\mathcal{G}, \mathcal{H}^{4}\right)_{\text {mult }} \rightarrow \operatorname{Inv}^{4}\left(\mathbf{G}, \mathcal{H}^{*}\right)
$$


Au total, on obtient un diagramme

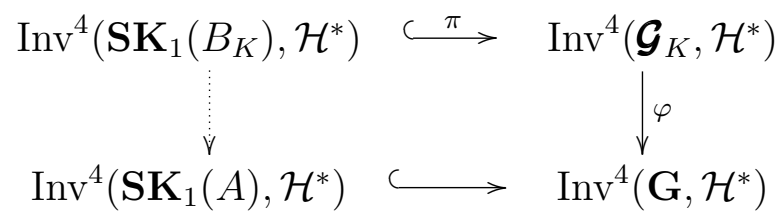

qui induit l'existence de la flêche pointée. En effet, soient $\rho \in \operatorname{Inv}^{4}\left(\operatorname{SK}_{1}\left(B_{K}\right), \mathcal{H}^{*}\right)$ et $(F, S, \bar{F})$ une extension de Cohen de $(K, R, k)$, alors $(\varphi \circ \pi(\rho))_{\bar{F}}$ envoie les commutateurs de $A_{\bar{F}}^{\times}$à 0 puisqu'il correspondent aux commutateurs de $B_{F}^{\times}$grâce à l'isomorphisme $\mathbf{S K}_{1}(A)(\bar{F}) \cong \mathbf{S K}_{1}\left(B_{K}\right)(F)$ (Corollaire 3.3 ).

Dans les Théorèmes 4.16 et 4.20 , nous avons aussi construit ce morphisme pointé de groupes d'invariants mais d'une façon plus explicite.

\subsection{Conjecture de Suslin}

Nous finissons ce texte donc par quelques remarques autour de la conjecture de Suslin. Commençons par rappeler la conjecture.

Conjecture 5.1 (Suslin [Sus1]). Soient $k$ un corps et $A$ une $k$-algèbre simple centrale. Alors, $\boldsymbol{S K}_{1}(A)=0$ si est seulement si ind $(A)$ est sans facteurs carrés.

Comme mentionnée dans l'introduction, c'est une question de nécessité, puisque Wang a démontré que pour une algèbre simple centrale $A$, le groupe de Whitehead réduit $\mathbf{S K}_{1}(A)$ est trivial si $\operatorname{ind}_{k}(A)$ est sans facteurs carrés [Wan]. Dû à la décomposition 4.9 , il suffit de traiter le cas où l'indice de $A$ est $p$-primaire pour un premier $p$. De plus, par la formule de réduction d'indice, il suffit de démontrer que $\mathbf{S K}_{1}(A) \neq 0$ si ind ${ }_{k}(A)=p^{2}(p$ premier $)$ Bla, Prop. 4].

Nous pouvons ajouter une question sur l'invariant de Suslin.

Question 5.2. Soient $k$ un corps et $A$ une $k$-algèbre simple centrale de ind $d_{k}(A)$ contenant un facteur carré. Est-ce que l'invariant de Suslin $\rho_{\text {Sus }, A}$ est non trivial?

Remarque 5.3 - Bien évidemment, une réponse affirmative à cette question impliquerait la conjecture de Suslin. Alors, on pourrait appeler cette question la version forte de la conjecture de Suslin.

De nouveau, par la formule de réduction de l'indice, il suffit de répondre à la question pour des $k$-algèbres simples centrales $A$ de $\operatorname{ind}_{k}(A)=p^{2}$ ( $p$ premier).

Merkurjev a démontré que la conjecture de Suslin vaut pour des algèbres simples centrales avec indice divisible par 4 (par exemple une $k$-algèbre de biquaternions) [Mer2]. Puisque l'invariant de Suslin pour des biquaternions est injectif dans le cas modéré Sus2, Thm. 3], il est par construction aussi injectif dans le cas sauvage. Alors, on obtient que pour une $k$-algèbre simple centrale $A$ de $\operatorname{ind}_{k}(A)$ divisible par $4, \rho_{\text {Sus, A }}$ n'est pas trivial, indépendamment de $\operatorname{car}(k)$ (en particulier pour les caractéristiques sauvages, comme 2).

Récemment Rehman-Tikhonov-Yanchevskiı̌ ont en plus démontré qu'il suffit de vérifier la conjecture de Suslin pour des algèbres à division cycliques. Il suffit même de démontrer la conjecture pour une classe d'algèbres à division cycliques élémentaires (des produits tensoriels de deux algèbres cycliques de Dickson) [RTY, Thm 0.19 - 0.20]. 
En utilisant des relèvements d'algèbres simples centrales de la caractéristique positive à la caractéristique 0 comme dans 3.2 (a), on obtient l'énoncé de comparaison suivant.

Proposition 5.4. Soient $(K, R, k)$ un p-triplet de Cohen, A une k-algèbre simple centrale et B la R-algèbre d'Azumaya relevée. Si la conjecture de Suslin (forte) vaut pour A, alors elle vaut aussi pour $B_{K}$.

Démonstration. Rappelons que $\operatorname{ind}_{k}(A)=\operatorname{ind}_{K}\left(B_{K}\right)$. L'enoncé sur la conjecture de Suslin même suit donc immédiatement du Corollaire 3.3. L'enoncé sur la conjecture de Suslin forte est vrai, parce que l'invariant de Suslin satisfait/est défini par un morphisme

$$
\operatorname{Inv}^{4}\left(\mathbf{S K}_{1}\left(B_{K}\right), \mathcal{H}_{r, L, \mathcal{B}_{K}}^{*}\right) \rightarrow \operatorname{Inv}^{4}\left(\mathbf{S K}_{1}(A), \mathcal{H}_{r, L, \mathcal{A}}^{*}\right)
$$

Remarque 5.5 - Une réciproque de la Proposition 5.4 est une question ouverte et ne suit pas formellement des définitions. En effet, si $\mathbf{S K}_{1}(A)=0$, c'est-à-dire $\operatorname{SK}_{1}\left(A \otimes_{k} k^{\prime}\right)=$ 0 pour toute extension de corps $k^{\prime}$ de $k$. Alors, $\operatorname{SK}_{1}\left(B_{K} \otimes_{K} K^{\prime}\right)=\operatorname{SK}_{1}\left(A \otimes_{k} k^{\prime}\right)=$ 0 pour toute extension de Cohen $\left(K^{\prime}, R^{\prime}, k^{\prime}\right)$ de $(K, R, k)$. Mais, il n'est pas sûr que $\mathrm{SK}_{1}\left(B_{K} \otimes_{K} F\right)=0$ pour toute extension $F$ de $K$. On peut reformuler la situation aussi dans le cadre de $\$ 5.1$; la Question 5.2 se traduit à la possible injectivité du morphisme (5.2).

Les constructions effectuées par l'auteur ne lui semblent donc pas donner de façons immédiates à faire des réductions fortes de caractéristiques. Il serait intéressant de pouvoir définir une des flèches pointées (dans un sens au choix) dans le diagramme en bas. On y abrège la conjecture de Suslin (forte) par CS(F).

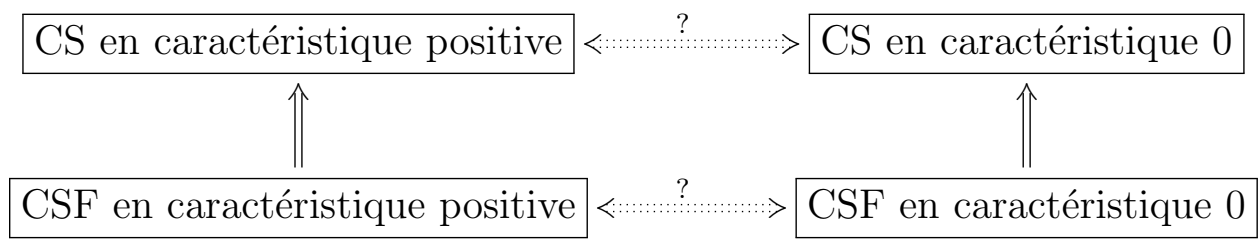

\section{A Vérification des règles}

Dans cette appendice, on vérifie que toutes les règles sont bien définies pour le module de cycles $\mathcal{H}_{p^{n}, L}^{*}$ de la Section 4 . Nous rappelons les règles pour un module de cycles $M$ de base $R$ et vérifions qu'ils vont pour le module de cycle $\mathcal{H}_{p^{n}, L}^{*}$. Dans les règles, $E, F, G$ sont des $R$-corps arbitraires, et toute application entre des corps est un morphisme de $R$-corps.

R1a : Pour tous $\varphi: F \rightarrow E, \psi: E \rightarrow G$, on a $(\psi \circ \varphi)_{*}=\psi_{*} \circ \varphi_{*}$.

R1b : Pour tous $\varphi: F \rightarrow E, \psi: E \rightarrow G$ finis, on a $(\psi \circ \varphi)^{*}=\varphi^{*} \circ \psi^{*}$.

R1c : Soient $\varphi: F \rightarrow E, \psi: F \rightarrow G$ avec $\varphi$ fini et $S=G \otimes_{F} E$. Pour $p \in \operatorname{Spec}(S)$, soient $\varphi_{p}: G \rightarrow S / p, \psi_{p}: E \rightarrow S / p$ les applications naturelles et $l_{p}$ la longueur de l'anneau localisé $S_{(p)}$. Alors,

$$
\psi_{*} \circ \varphi^{*}=\sum_{p} l_{p} \cdot\left(\varphi_{p}\right)^{*} \circ\left(\varphi_{p}\right)_{*}
$$


R2 : Pour $\varphi: F \rightarrow E, x \in K_{*} F, y \in K_{*} E, \rho \in M(F), \mu \in M(E)$, on a (avec $\varphi$ fini dans $\mathrm{R} 2 \mathrm{~b}$ et $\mathrm{R} 2 \mathrm{c})$ :

$\mathbf{R 2 a}: \varphi_{*}(x \cdot \rho)=\varphi_{*}(x) \cdot \varphi_{*}(\rho)$,

$\mathbf{R 2 b}: \varphi^{*}\left(\varphi_{*}(x) \cdot \mu\right)=x \cdot \varphi^{*}(\mu)$, et

R2c : $\varphi^{*}\left(y \cdot \varphi_{*}(\rho)\right)=\varphi^{*}(y) \cdot \rho$.

R3a : Soient $\varphi: E \rightarrow F$ et $v$ une valuation de $F$ qui se restreint à une valuation $w$ non triviale sur $E$ avec indice de ramification $e$. Soit $\bar{\varphi}: \kappa(w) \rightarrow \kappa(v)$ l'application induite. Alors,

$$
\partial_{v} \circ \varphi_{*}=e \cdot \bar{\varphi}_{*} \circ \partial_{w} .
$$

R3b : Soient $\varphi: F \rightarrow E$ fini et $v$ une valuation de $F$. Pour toute extension $w$ de $v$ sur $E$, soit $\varphi_{w}: \kappa(v) \rightarrow \kappa(w)$ l'application induite. Alors,

$$
\partial_{v} \circ \varphi^{*}=\sum_{w} \varphi_{w}^{*} \circ \partial_{w}
$$

R3c : Soient $\varphi: E \rightarrow F$ et $v$ une valuation de $F$ qui est triviale sur $E$. Alors,

$$
\partial_{v} \circ \varphi_{*}=0
$$

R3d : Soient $\varphi: E \rightarrow F, v$ une valuation de $F$ qui est triviale sur $E, \bar{\varphi}: E \rightarrow \kappa(v)$ l'application induite et $\pi$ une uniformisante de $v$. Soit de plus $s_{v}^{\pi}: M(F) \rightarrow$ $M(\kappa(v))$ défini par $s_{v}^{\pi}(\rho)=\partial_{v}(\{-\pi\} \cdot \rho)$, alors

$$
s_{v}^{\pi} \circ \varphi_{*}=\bar{\varphi}_{*} .
$$

R3e : Soient $v$ une valuation sur $F, u$ une $v$-unité et $\rho \in M(F)$, alors on a

$$
\partial_{v}(\{-u\} \cdot \rho)=-\{\bar{u}\} \cdot \partial_{v}(\rho) .
$$

Pour un $R$-schéma $\mathcal{X}$, on note $M(x)=M(\kappa(x))$ pour $x \in \mathcal{X}$. Si $\mathcal{X}$ est irréductible, son point générique est noté $\xi$. Si $\mathcal{X}$ est normal, tout $x \in \mathcal{X}^{(1)}$ induit $\partial_{x}: M(\xi) \rightarrow M(x)$. Pour tous $x, y \in \mathcal{X}$, on définit maintenant $\partial_{y}^{x}$. On pose $\partial_{y}^{x}=0$ si $Z=\overline{\{x\}}$ et $y \notin Z^{(1)}$. Autrement, soit $\tilde{Z} \rightarrow Z$ la normalisation et

$$
\partial_{y}^{x}:=\sum_{z \mid y} \varphi_{z}^{*} \circ \partial_{z}
$$

où $z$ parcourt les points de $\tilde{Z}$ au dessus de $y$ et $\varphi_{z}$ est le morphisme fini $\kappa(y) \rightarrow \kappa(z)$.

FD : ( "Finite support of divisors ») Soient $\mathcal{X}$ un $R$-schéma normal et $\rho \in M(\xi)$. Alors, $\partial_{x}(\rho)=0$ pour presque tout $x \in \mathcal{X}^{(1)}$.

C : ( Closedness ») Soient $\mathcal{X}$ intégral, local de dimension 2 et $x_{0}$ le point fermé de $\mathcal{X}$. Alors,

$$
0=\sum_{x \in \mathcal{X}^{(1)}} \partial_{x_{0}}^{x} \circ \partial_{x}^{\xi}: M(\xi) \rightarrow M\left(x_{0}\right) .
$$

Proposition A.1. Soit $(K, R, k)$ un p-triplet de Cohen avec $(L, S, \bar{L})$ une extension de Cohen finie galoisienne. Alors, $\mathcal{H}_{p^{n}, L}^{*}$ de la Définition 4.3 respecte les règles $R 1 a-R 3 e, F D$ et $C$ de module de cycles $(n>1)$ un entier. 
Remarque A.2 - Les données D1-D4 sont données dans \$4.1 (b), (d), (e) et (f).

Démonstration. Les règles $\mathrm{R} 1 \mathrm{a}-\mathrm{R} 3 \mathrm{e}$ suivent immédiatement de la définition de $\mathcal{H}_{p^{n}, L}^{*}$. À noter que la règle $\mathrm{R} 1 \mathrm{c}$ suit de la propriéte universelle des produits tensoriels. La vérification de la règle FD suit comme dans le cas classique du support fini des diviseurs [Har, Ch. II. Lem. 6.1].

Nous allons déduire la règle $C$ du fait qu'il vaut pour les $K$-groupes de Milnor Kat2. Les résidus $\partial_{K}$ pour les $K$-groupes de Milnor sont expliqués dans $\$ 2.1$ (d) et dans (4.7). Pour éviter une $K$-cophonie, nous supposons pour cette partie que la base de module de cycles est $(F, R, \bar{F})$ au lieu de $(K, R, k)$. Soit donc $\mathcal{X}$ un $R$-schéma intégral et local de dimension 2. On suppose tout d'abord que le morphisme structural $\mathcal{X}$ est surjectif. Alors, $X:=\mathcal{X} \times{ }_{R} F$ est un $F$-schéma et $Y:=\mathcal{X} \times{ }_{R} \bar{F}$ est un $\bar{F}$-schéma, tous les deux de dimension 1 et $\operatorname{car}(F(X))=0$ et $\operatorname{car}(\bar{F}(Y))=p$. Il faut donc vérifier que la composition des résidus fournit un complexe ( $y_{0}$ le point fermé de $\mathcal{X}$ et $\left.q \geq 2\right)$ :

$$
H_{p^{n}, L}^{q+1}(F(X)) \rightarrow \bigoplus_{x \in X^{(1)}} H_{p^{n}, L}^{q}(F(x)) \oplus \bigoplus_{y \in Y^{(0)}} H_{p^{n}, L}^{q}(\bar{F}(y)) \rightarrow H_{p^{n}, L}^{q-1}\left(\bar{F}\left(y_{0}\right)\right)
$$

Nous allons décrire les groupes et les résidus en question avec des $K$-groupes pour pouvoir utiliser la règle $C$ pour les groupes de $K$-théorie. Décrivons d'abord les différents groupes avec la $K$-théorie de Milnor.

- Le groupe $H_{p^{n}, L}^{q+1}(F(X))$ :

Puisque

$$
\Gamma=\operatorname{Gal}\left(F_{\mathrm{nr}}(X) / F(X)\right) \cong \operatorname{Gal}\left(F_{\mathrm{nr}} / F\right) \cong \operatorname{Gal}\left(\bar{F}_{s} / \bar{F}\right),
$$

on sait que $\operatorname{cd}_{p}(\Gamma) \leq 1$ [Ser2, Ch. II, Prop. 3]. La suite spectrale de Hochschild-Serre

$$
E_{2}^{s, t}:=H^{s}\left(\Gamma, H^{t}\left(F_{\mathrm{nr}}(X), \mu_{p^{n}}^{\otimes q}\right)\right) \Longrightarrow H^{s+t}\left(F(X), \mu_{p^{n}}^{\otimes q}\right)
$$

induit donc un isomorphisme

$$
H^{1}\left(\Gamma, H^{q}\left(F_{n r}(X), \mu_{p^{n}}^{\otimes q}\right)\right) \cong \operatorname{ker}\left[H_{p^{n}}^{q+1}(F(X)) \rightarrow H_{p^{n}}^{q+1}\left(F_{\mathrm{nr}}(X)\right)\right] .
$$

La conjecture de Bloch-Kato, prouvée par Voevodsky-Rost-Weibel [BK, Voe, Ros2, Wei], dit en plus que $H^{q}\left(F_{\mathrm{nr}}(X), \mu_{p^{n}}^{\otimes q}\right) \cong K_{q}\left(F_{\mathrm{nr}}(X)\right) / p^{n}$. Ceci nous donne un isomorphisme

$$
H^{1}\left(\Gamma, K_{q}\left(F_{\mathrm{nr}}(X)\right) / p^{n}\right) \cong \operatorname{ker}\left[H_{p^{n}}^{q+1}(F(X)) \rightarrow H_{p^{n}}^{q+1}\left(F_{\mathrm{nr}}(X)\right)\right]
$$

et donc une inclusion

$$
H_{p^{n}, L}^{q+1}(F(X)) \subset H^{1}\left(\Gamma, K_{q}\left(F_{\mathrm{nr}}(X)\right) / p^{n}\right) .
$$

- Le groupe $H_{p^{n}, L}^{q+1}(F(x))$ pour $x \in X^{(1)}$ :

De même façon que ci-dessus, on obtient une inclusion

$$
H_{p^{n}, L}^{q}(F(x)) \subset H^{1}\left(\Gamma, K_{q-1}\left(F_{\mathrm{nr}}(x)\right) / p^{n}\right) .
$$

- Le groupe $H_{p^{n}, L}^{q}(\bar{F}(y))$ pour $y \in Y^{(0)}$ :

Soit $y \in Y^{(0)}$, alors $H_{p^{n}}^{q}(\bar{F}(y)) \cong H^{1}\left(\bar{F}(y), \nu_{n}(q-1)_{\bar{F}(y)_{s}}\right)$. L'isomorphisme de 
Bloch-Kato-Gabber $\nu_{n}(q-1)_{\bar{F}(y)_{s}} \cong K_{q-1}\left(\bar{F}(y)_{s}\right) / p^{n}[\mathrm{BK}$, Thm. 2.1] donne donc un isomorphisme,

$$
H^{1}\left(\bar{F}(y), K_{q-1}\left(\bar{F}(y)_{s}\right) / p^{n}\right) \cong H_{p^{n}}^{q+1}(\bar{F}(y)),
$$

qui implique aussi une inclusion :

$$
\begin{aligned}
H_{p^{n}, L}^{q}(\bar{F}(y)) & \cong \operatorname{ker}\left[H^{1}\left(\bar{F}(y), K_{q-1}\left(\bar{F}(y)_{s}\right) / p^{n}\right) \rightarrow H^{1}\left(\bar{L}(y), K_{q-1}\left(\bar{F}(y)_{s}\right) / p^{n}\right)\right] \\
& \subset \operatorname{ker}\left[H^{1}\left(\bar{F}(y), K_{q-1}\left(\bar{F}(y)_{s}\right) / p^{n}\right) \rightarrow H^{1}\left(\bar{F}_{s}(y), K_{q-1}\left(\bar{F}(y)_{s}\right) / p^{n}\right)\right] .
\end{aligned}
$$

Ce dernier est isomorphe à $H^{1}\left(\Gamma,\left(K_{q-1}\left(\bar{F}(y)_{s}\right) / p^{n}\right)^{\Gamma_{\bar{F}_{s}(y)}}\right)$ par la suite d'inflationrestriction [GS, Prop. 3.3.14].

- Le groupe $H_{p^{n}, L}^{q}\left(\bar{F}\left(y_{0}\right)\right)$ pour yo le point fermé de $\mathcal{X}$ :

Alors comme au-dessus :

$$
H_{p^{n}, L}^{q-1}\left(\bar{F}\left(y_{0}\right)\right) \subset H^{1}\left(\Gamma,\left(K_{q-2}\left(\bar{F}\left(y_{0}\right)_{s}\right) / p^{n}\right)^{\Gamma_{\bar{F}}\left(y_{0}\right)}\right) .
$$

Expliquons maintenant les résidus en termes de $K$-théorie.

- Le résidu $\partial_{x}: H_{p^{n}, L}^{q+1}(F(X)) \rightarrow H_{p^{n}, L}^{q+1}(F(x))$ pour $x \in X^{(1)}$ :

La valuation associée à $x$ induit bien le résidu $\partial_{x}$, mais aussi un résidu $\Gamma$-équivariant $\partial_{K, x}: K_{q}\left(F_{\mathrm{nr}}(X)\right) / p^{n} \rightarrow K_{q-1}\left(F_{\mathrm{nr}}(x)\right) / p^{n}$ (puisque $\operatorname{Gal}\left(F_{\mathrm{nr}}(x) / F(x)\right) \cong \Gamma$ ). Ceci induit donc un morphisme (à qui on donne le même nom par abus de notation) :

$$
\partial_{K, x}: H^{1}\left(\Gamma, K_{q}\left(F_{\mathrm{nr}}(X)\right) / p^{n}\right) \rightarrow H^{1}\left(\Gamma, K_{q-1}\left(F_{\mathrm{nr}}(x)\right) / p^{n}\right) .
$$

Le Lemme A.3 plus loin induit que $\partial_{K, x}$ est compatible avec $\partial_{x}$ par les inclusions (A.3) et (A.4), c'est-à-dire on a un diagramme commutatif :

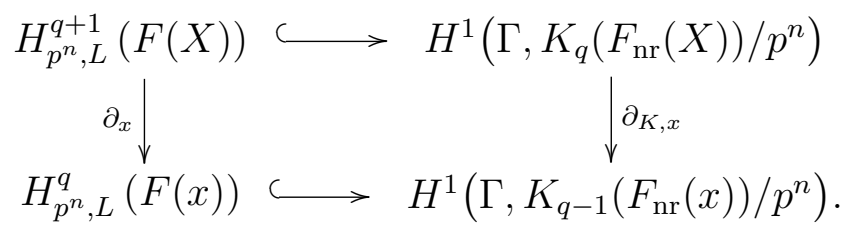

- Le résidu $\partial_{y}: H_{p^{n}, L}^{q+1}(F(X)) \rightarrow H_{p^{n}, L}^{q}(\bar{F}(y))$ pour $y \in Y^{(0)}$ :

La valuation associée à $y$ induit bien un résidu $\partial_{y}$. Dans le Lemme A.3, on démontre que sous l'injection $\mathrm{A} .5 \mathrm{im}\left(\partial_{y}\right)$ est envoyé dans $H^{1}\left(\Gamma, K_{q}\left(\bar{F}_{s}(y)\right) / p^{n}\right)$. De l'autre côté, la valuation associée à $y$ induit un résidu $\Gamma$-équivariant $\partial_{K, y}: K_{q}\left(F_{\mathrm{nr}}(X)\right) \rightarrow$ $K_{q-1}\left(\bar{F}_{s}(y)\right)$ et donc un morphisme :

$$
\partial_{K, y}: H^{1}\left(\Gamma, K_{q}\left(F_{\mathrm{nr}}(X)\right) / p^{n}\right) \rightarrow H^{1}\left(\Gamma, K_{q-1}\left(\bar{F}_{s}(y)\right) / p^{n}\right) .
$$

Le Lemme A.3 démontre qu'on a un diagramme commutif qui exprime la compatibilité de $\partial_{y}$ et de $\partial_{K, y}$ sous les inclusions (A.3) et A.5 :

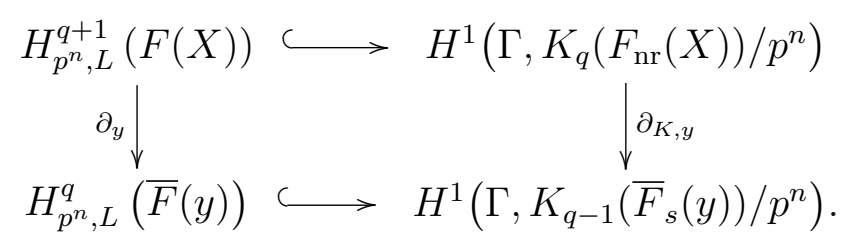


- Le résidu $\partial_{y_{0}}^{x}: H_{p^{n}, L}^{q+1}(F(x)) \rightarrow H_{p^{n}, L}^{q+1}\left(F\left(y_{0}\right)\right)$ pour $x \in X^{(1)}$ :

De nouveau, on a bien le résidu $\partial_{y_{0}}^{x}$, et dans le Lemme A.3 on démontre que sous l'inclusion A.6 $\operatorname{im}\left(\partial_{y_{0}}^{x}\right)$ est contenu dans $\left.H^{1}\left(\Gamma, K_{q-2}\left(\overline{F_{s}\left(y_{0}\right.}\right)\right) / p^{n}\right)$. Par ailleurs, on a aussi un résidu $\Gamma$-équivariant $\partial_{K, y_{0}}^{x}: K_{q-1}\left(F_{\mathrm{nr}}(x)\right) \rightarrow K_{q-2}\left(\bar{F}_{s}\left(y_{0}\right)\right)$ qui donne au niveau de cohomologie un morphisme :

$$
\partial_{K, y_{0}}^{x}: H^{1}\left(\Gamma, K_{q-1}\left(F_{\mathrm{nr}}(x)\right) / p^{n}\right) \rightarrow H^{1}\left(\Gamma, K_{q-2}\left(\bar{F}_{s}\left(y_{0}\right)\right) / p^{n}\right) .
$$

De nouveau, le Lemme A.3 garantit que $\partial_{K, y_{0}}^{x}$ est compatible avec $\partial_{y_{0}}^{x}$ sous les inclusions A.4 et A.6 formant ainsi le diagramme commutatif :

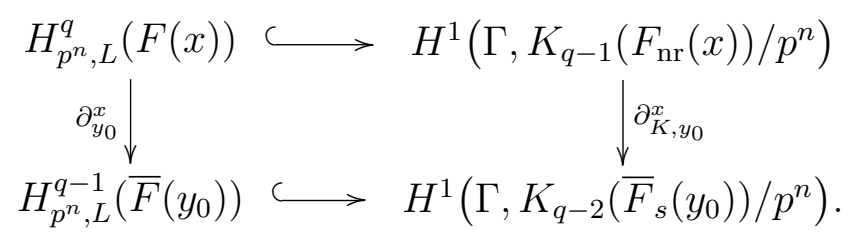

- Le résidu $\partial_{y_{0}}^{y}: H_{p^{n}, L}^{q}(\bar{F}(y)) \rightarrow H_{p^{n}, L}^{q+1}\left(F\left(y_{0}\right)\right)$ pour $y \in Y^{(0)}:$

Dans cette situation, on a aussi un résidu $\partial_{y_{0}}^{y}$ sur les groupes de cohomologie et un résidu $\Gamma$-équivariant de la $K$-théorie $\partial_{K, y_{0}}^{y}: K_{q-1}\left(\bar{F}_{s}(y)\right) \rightarrow K_{q-2}\left(\bar{F}_{s}\left(y_{0}\right)\right)$ (pour $\left.y \in Y^{(1)}\right)$. Alors, $\partial_{K, y_{0}}^{y}$ induit un morphisme au niveau de cohomologie :

$$
\partial_{K, y_{0}}^{y}: H^{1}\left(\Gamma, K_{q-1}\left(\bar{F}_{s}(y)\right) / p^{n}\right) \rightarrow H^{1}\left(\Gamma, K_{q-2}\left(\bar{F}_{s}\left(y_{0}\right)\right) / p^{n}\right),
$$

et le Lemme A.3 démontre la compatibilité de $\partial_{K, y_{0}}^{y}$ avec $\partial_{y_{0}}^{y}$ sous les inclusions A.5 et (A.6) :

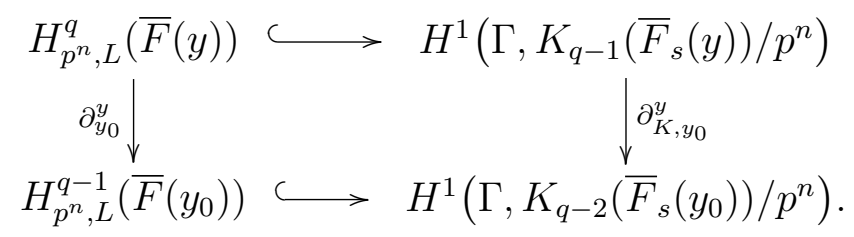

En somme, on a donc un ensemble de résidus,

$$
\begin{aligned}
H^{1}\left(\Gamma, K_{q}\left(F_{\mathrm{nr}}(X)\right) / p^{n}\right) \rightarrow \bigoplus_{x \in X^{(1)}} H^{1}\left(\Gamma, K_{q-1}\left(F_{\mathrm{nr}}(x)\right) / p^{n}\right) \oplus & \bigoplus \bigoplus_{y \in Y^{(0)}} H^{1}\left(\Gamma, K_{q-1}\left(\bar{F}_{s}(y)\right) / p^{n}\right) \\
& \rightarrow H^{1}\left(\Gamma, K_{q-2}\left(\bar{F}_{s}\left(y_{0}\right)\right) / p^{n}\right)
\end{aligned}
$$

dont on sait que c'est un complexe parce que les $K$-groupes de Milnor respectent la règle

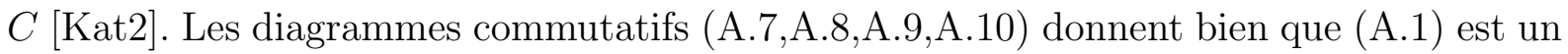
complexe.

Si le morphisme structural n'est pas surjectif, on a ou bien un $F$-schéma, ou bien un $\bar{F}$-schéma. Si $\mathcal{X}$ est un $F$-schéma, les groupes en vigueur sont définis commes des noyaux des groupes de la cohomologie galoisienne modéré. La règle $C$ suit donc de la règle $C$ du cas modéré. Si $\mathcal{X}$ est un $\bar{F}$-schéma, on réécrit A.1 avec (4.2) et l'isomorphisme de Bloch-Gabber-Kato comme

$$
H^{1}\left(\Gamma, K_{q}\left(\bar{F}_{s}(\mathcal{X})\right) / p^{n}\right) \rightarrow \bigoplus_{x \in \mathcal{X}^{(1)}} H^{1}\left(\Gamma, K_{q-1}\left(\bar{F}_{s}(x)\right) / p^{n}\right) \rightarrow H^{1}\left(\Gamma, K_{q-1}\left(\bar{F}_{s}\left(x_{0}\right)\right) / p^{n}\right),
$$

avec $x_{0}$ le point fermé de $\mathcal{X}$. Celui est de nouveau un complexe, parce que les résidus sont compatibles avec les résidus de la $K$-théorie de Milnor (voir Lemme A.3 dans le cas " $y$ et $\left.y_{0}{ }^{\prime \prime}\right)$, et parce que la règle $C$ vaut pour la $K$-théorie de Milnor [Kat2]. 
Lemme A.3. Soit $\mathcal{X}$ un $R$-schéma tel que le morphisme structural est surjectif, alors les diagrammes $A .7$ A.8 A.9 A.10) sont commutatifs.

Démonstration. On a quatre situations; traitons-les cas par cas.

- Le diagramme (A.7) est commutatif pour $x \in X^{(1)}$ :

L'isomorphisme $K_{q}\left(F_{n r}(X)\right) / p^{n} \cong H^{q}\left(F_{\mathrm{nr}}(X), \mu_{p^{n}}^{\otimes q}\right)$ de Bloch-Kato est défini par le symbole galoisien. Cet isomorphisme commute avec le résidu usuel sur $H^{q}\left(F_{\mathrm{nr}}(X), \mu_{p^{n}}^{\otimes q}\right)$ (avec une section donnée par le cup-produit par la classe d'une uniformisante $\pi_{x}$ pour la valuation associée à $x$ ) [GS, Prop. 7.5.1]. On en déduit le résultat, puisque l'isomorphisme A.2 est l'inflation, et puisque $\partial_{x}$ a aussi une section donnée par le cup-produit par la classe de $\pi_{x}$.

- Le diagramme (A.8) est commutatif pour $y \in Y^{(0)}$ :

Il faut donc aussi vérifier que $\operatorname{im}\left(\partial_{y}\right)$ est contenu dans $H^{1}\left(\Gamma, K_{q-1}\left(\bar{F}_{s}(y)\right) / p^{n}\right)$. Parce que le résidu $\partial_{y}$ est défini par une section, on peut prendre $w \otimes \bar{x}_{2} \otimes \ldots \otimes \bar{x}_{q} \in$ $H_{p^{n}, L}^{q}(\bar{F}(y))$ avec $w \in W_{n}(\bar{F}(y))$ et $x_{2}, \ldots, x_{q} \in \mathcal{O}_{y}^{\times}\left(\mathcal{O}_{y}\right.$ étant l'anneau de valuation associé à la valuation induite par $y)$. Si $\pi_{y}$ est une uniformisante de $F(X)$ pour la valuation associée à $y$, il est le résidu de

$$
i(w) \cup h_{p^{n}, F(X)}^{q}\left(\left\{\pi_{y}, x_{2}, \ldots x_{q}\right\}\right) \in H_{p^{n}, L}^{q+1}(F(X)) .
$$

Il correspond donc à

$$
\left((\sigma(a)-a)\left\{\pi_{y}, x_{2}, \ldots, x_{q}\right\}\right)_{\sigma} \in H^{1}\left(\Gamma, K_{q}\left(F_{\mathrm{nr}}(X)\right) / p^{n}\right),
$$

où $a^{(p)}-a=w$ avec $a \in W_{n}(\bar{F}(y))$ et où on considère $(\sigma(a)-a)$ comme élement de $\mathbb{Z} / p^{n} \mathbb{Z}$. Par ailleurs, $w \otimes \bar{x}_{2} \otimes \ldots \otimes \bar{x}_{q}$ correspond à

$$
\left((\sigma(a)-a)\left\{\bar{x}_{2}, \ldots, \bar{x}_{q}\right\}\right)_{\sigma} \in H^{1}\left(\Gamma, K_{q}\left(\bar{F}(y)_{s}\right) / p^{n}\right) .
$$

La commutativité suit donc, et il est aussi clair que $\left((\sigma(a)-a)\left\{\bar{x}_{2}, \ldots, \bar{x}_{q}\right\}\right)_{\sigma}$ est en effet un élément de $H^{1}\left(\bar{F}(y), K_{q}\left(\bar{F}_{s}(y)\right) / p^{n}\right)$, parce que $\partial_{K, y}$ tombe dedans.

- Le diagramme (A.9) est commutatif pour $x \in X^{(1)}$ :

On sait le vérifier de façon analogue au cas précedent.

- Le diagramme A.10 est commutatif pour $y \in Y^{(0)}$ :

Les isomorphismes

$$
\nu_{n}(q-1)_{\bar{F}(y)_{s}} \cong K_{q-1}\left(\bar{F}(y)_{s}\right) / p^{n}, \quad \nu_{n}(q-2)_{\bar{F}\left(y_{0}\right)_{s}} \cong K_{q-2}\left(\bar{F}\left(y_{0}\right)_{s}\right) / p^{n},
$$

et le résidu $K_{q-1}\left(\bar{F}(y)_{s}\right) \rightarrow K_{q-2}\left(\bar{F}\left(y_{0}\right)_{s}\right)$ induisent un résidu,

$$
\begin{aligned}
\nu_{n}(q-1)_{\bar{F}(y)_{s}} & \rightarrow \nu_{n}(q-2)_{\bar{F}\left(y_{0}\right)_{s}, \quad \text { defini par }} \\
a \otimes \pi_{0} \otimes x_{2} \otimes \ldots \otimes x_{q-1} & \mapsto \bar{a} \otimes \bar{x}_{2} \ldots \otimes \bar{x}_{q-1} .
\end{aligned}
$$

Ici, $a \in W_{n}\left(\mathcal{O}_{v}\right)$ et $x_{i} \in \mathcal{O}_{v}^{\times}$, où $\mathcal{O}_{v}$ est l'anneau de valuation associé à la valuation $v$ induite par $y_{0}$ avec $\pi_{0}$ une uniformisante. Par la définition du résidu $\partial_{y_{0}}^{y}$ (voir les Remarques 4.2 et 4.7), il est clair que les résidus sont compatibles. 


\section{Références}

[AG] Maurice Auslander et Oscar Goldman. The Brauer group of a commutative ring. Trans. Amer. Math. Soc. 97, 367-409, 1960.

[Alb] Adrian Albert. Simple algebras of degree $p^{e}$ over a centrum of characteristic $p$. Trans. Amer. Math. Soc. 40(1), 112-126, 1936.

[BK] Spencer Bloch et Kazuya Kato. p-adic étale cohomology. Publ. Math. Inst. Hautes Études Sci. (63), 107-152, 1986.

[Bla] Altha Blanchet. Function fields of generalized Brauer-Severi varieties. Comm. Algebra, 19(1), 97-118, 1991.

[BT] Hyman Bass et John Tate. The Milnor ring of a global field. In Algebraic K-theory, II : "Classical" algebraic K-theory and connections with arithmetic (Proc. Conf., Seattle, Wash., Battelle Memorial Inst., 1972), pages 349-446. Lecture Notes in Math., Vol. 342. Springer, Berlin, 1973.

[Car] Pierre Cartier. Questions de rationalité des diviseurs en géométrie algébrique. Bull. Soc. Math. France, 86, 177-251, 1958.

[Coh] Irvin Cohen. On the structure and ideal theory of complete local rings. Trans. Amer. Math. Soc., 59, 54-106, 1946.

[EKLV] Hélène Esnault, Bruno Kahn, Marc Levine, et Eckart Viehweg. The Arason invariant and mod 2 algebraic cycles. J. Amer. Math. Soc., 11(1), 73-118, 1998.

[Gil1] Philippe Gille. Invariants cohomologiques de Rost en caractéristique positive. K-Theory, 21, 57-100, 2000.

[Gil2] Philippe Gille. Le problème de Kneser-Tits. Séminaire Bourbaki no 983, à paraître à Astérisque, 2009.

[GMS] Skip Garibaldi, Alexander Merkurjev, et Jean-Pierre Serre. Cohomological invariants in Galois cohomology, volume 28 de University Lecture Series. Amer. Math. Soc., 2003.

[Gro1] Alexander Grothendieck. Éléments de Géométrie Algébrique IV, Étude locale des schémas et des morphismes de schémas, Première Partie, volume 20 de Publ. Math. Inst. Hautes Études Sci. Bures-sur-Yvette, 1964.

[Gro2] Alexander Grothendieck. Le groupe de Brauer : I. Algèbres d'Azumaya et interprétations diverses. Séminaire Bourbaki, 9, 199-219, 1964-1966. Exposé No. 290.

[GS] Philippe Gille et Tamás Szamuely. Central Simple Algebras and Galois Cohomology, volume 101 de Cambridge studies in advanced mathematics. Cambridge University Press, Cambridge, 2006.

[Har] Robin Hartshorne. Algebraic Geometry, volume 52 de Graduate Texts in Mathematics. Springer Science+Business Media, Inc., New York, 1977.

[Izh] Oleg Izhboldin. On the cohomology groups of the field of rational functions. In Mathematics in St. Petersburg, volume 174 de Amer. Math. Soc. Transl. Ser. 2, pages 21-44. Amer. Math. Soc., Providence, RI, 1996.

[Kah] Bruno Kahn. Applications of weight-two motivic cohomology. Doc Math. J. DMV, 1, 395-416, 1996.

[Kat1] Kazuya Kato. Galois cohomology of complete discrete valuation fields. In Algebraic K-Theory, volume 967 de Lecture notes in mathematics, pages 215-238, Berlin, 1982.

[Kat2] Kazuya Kato. A Hasse principle for two-dimensional global fields. J. Reine Angew. Math., 366, 142-183, 1986.

[KMRT] Max-Albert Knus, Alexander Merkurjev, Markus Rost, et Jean-Pierre Tignol. The book of involutions, volume 44 de Amer. Math. Soc. Colloq. Publ. 1998.

[Knu] Max-Albert Knus. Quadratic and Hermitian forms over rings, volume 294 de Grundlehren der Mathematischen Wissenschaften. Springer-Verlag, Berlin, 1991.

[Lic] Stephen Lichtenbaum. The construction of weight-two arithmetic cohomology. Invent. math. 88, 183-215, 1987. 
[Mer1] Alexander Merkurjev. Invariants of algebraic groups. J. reine angew. Math. 508, 127-156, 1999.

[Mer2] Alexander Merkurjev. The group $S K_{1}$ for simple algebras. K-Theory, 37(3), 311-319, 2006.

[Mil] John Milnor. Algebraic K-theory and quadratic forms. Invent. Math. 9, 318-344, 1969/1970.

[NM] Tadasi Nakayama et Yozô Matsushima. Über die multiplikative Gruppe einer $p$-adischen Divisionsalgebra. Proc. Imp. Acad. Tokyo, 19, 622-628, 1943.

[Pan] Ivan Panin. Splitting principle and $K$-theory of simply connected semisimple algebraic groups. Algebra i Analiz, 10(1), 88-131, 1998.

[Pla] Vladimir Platonov. The Tannaka-Artin problem and reduced K-theory. Math. USSR Izv., 10(2), 211-243, 1976. Traduction anglaise.

[Ros1] Markus Rost. Chow Groups with Coefficients. Doc. Math. J. DMV, 1, 319-393, 1996.

[Ros2] Markus Rost. The basic correspondence of a splitting variety. Notes téléchargables de son site personnel, 1998.

[RTY] Ulf Rehman, Sergey Tikhonov, et Vyacheslav Yanchevskiu. Symbols and cyclicity of algebras after a scalar extension. Preprint, 2008.

[San] Jean-Jacques Sansuc. Groupe de Brauer et arithmétique des groupes algébriques linéaires. J. reine angew. Math., 327, 12-80, 1981.

[Sch] Colette Schoeller. Groupes affines, commutatifs, unipotents sur un corps parfait. Bulletin de la S.M.F., 100, 241-300, 1972.

[Ser1] Jean-Pierre Serre. Corps Locaux. Publications de l'Institut de Mathématique de l'Université de Nancago. Hermann, Paris, 1968.

[Ser2] Jean-Pierre Serre. Galois Cohomology. Springer Monographs in Mathematics. Springer-Verlag, Berlin, 2002.

[Sus1] Andrei Suslin. $S K_{1}$ of division algebras and Galois cohomology. In Algebraic K-theory, volume 4 de Adv. Soviet Math., pages 75-99. Amer. Math. Soc., Providence, RI, 1991.

[Sus2] Andrei Suslin. $S K_{1}(A)$ of division algebras and Galois cohomology revisited, volume 219 de Math. Soc. Transl. Ser. 2, pages 125-147. Am. Math. Soc., 2006.

[Voe] Vladimir Voevodsky. On Motivic Cohomology with $\mathbb{Z} / l$ coefficients. Prépublication, 2003.

[Wad] Adrian Wadsworth. Valuation theory on finite dimensional division algebras. In Valuation theory and its applications, Vol. I (Saskatoon, SK, 1999), volume 32 de Fields Inst. Commun., pages 385-449. Amer. Math. Soc., Providence, RI, 2002.

[Wan] Shianghaw Wang. On the commutator group of a simple algebra. Amer. J. Math., 72, 323-334, 1950 .

[Wei] Charles Weibel. 2007 Trieste Lectures on The Proof of the Bloch-Kato Conjecture, volume 23 de ICTP Lecture Notes Series, pages 1-28. 2008.

[Wit] Ernst Witt. Zyklische Körper und Algebren der Charakteristic $p$ vom Grad $p^{n}$. J. reine angew. Math. 176, 126-140, 1937.

[Wou] Tim Wouters. On Suslin's invariant for biquaternion algebras. En préparation, 2009. 